\title{
COMPARACIÓN DE DOS MÉTODOS DE MUESTREO VISUAL DE PECES DE ARRECIFES CORALINOS EN LA BAHÍA DE MURDEIRA, ISLA DE SAL, CABO VERDE
}

\author{
Isabel Sancibrián*, Rui Freitas** y José Carlos Hernández***
}

\section{RESUMEN}

Este estudio pretende comparar la eficacia dos métodos visuales de muestreo subacuático en las comunidades de peces tropicales de la Bahía de Murdeira, en la costa suroeste de la Isla de Sal, Cabo Verde. Se contrastaron dos tipos de métodos, el de transecto y el de punto fijo o circular. Los parámetros valorados fueron la riqueza y la abundancia de especies así como la diversidad (d'). Los resultados muestran diferencias significativas en la interacción entre el método y la localidad, siendo solamente la Localidad 2 aquella que mostró diferencias significativas en las estimas generadas por cada método. El análisis de las especies muestreadas realizado para todo el experimento pone de manifiesto que el método circular es el más completo, ya que es capaz de registrar mayor número de especies y de individuos que el método del transecto. Por último, el análisis de la diversidad revela que la localidad 2 es la más diversa, coincidiendo con la única localidad donde se encontraron diferencias en las estimas según el método de muestreo utilizado. Con este estudio se pretende resaltar las ventajas de la utilización de metodologías no destructivas y la importancia de tener en cuenta las características de la zona de estudio a la hora de elegir el método de muestreo.

Palabras clave: métodos visuales subacuáticos, peces tropicales, transecto, punto fijo, abundancia, diversidad, riqueza, no destructivas.

A COMPARISON OF TWO VISUAL CENSUS METHODS OF CORAL REEF FISHES IN THE BAHÍA DE MURDEIRA, SAL ISLAND, CABO VERDE

\section{Abstract}

This study aims to compare the efficiency of two underwater visual census methods on tropical fish communities of Murdeira bay, off the southwestern coast of Sal Island, Cabo Verde. Two methods were tested: strip belt transect and stationary point count. Parameters compared were: species richness, abundance and diversity (d'). The results show that there are significant differences due to the interaction between the method and location, being site 2 the one that showed significant differences in the estimates generated by each method. The analysis of species sampled performed for the entire experiment shows that the stationary point count method is the most complete, as it is able to record more species and individuals than the transect method. Finally, the diversity analysis reveals that site 2 is the most diverse, coinciding with the only location where differences in the estimates were found according to the sampling method used. This study highlight the advantages of using non-destructive methods and the importance of taking into account the characteristics of the study area for choosing the sampling method.

KEYwORDs: underwater visual census methods, reef fish, transect, stationary point count, abundance, diversity, richness, non-destructive. 


\section{INTRODUCCIÓN}

Los métodos de censo visual subacuáticos han sido ampliamente utilizados en estudios sobre dinámica de poblaciones y ecología de peces (Barans y Bortone 1983; Longo y Floeter 2012). Tanto en zonas templadas como en tropicales, estas técnicas son fundamentales para realizar un estudio preciso de la ictiofauna de un lugar (Harmelin-Vivien et al. 1985). Estos censos permiten estimar correctamente la abundancia y tallas de peces litorales. Sin embargo, y a pesar de que existen varios estudios que comparan experimentalmente los resultados producidos por algunos de los métodos más comunes de censo visual (Harmelin-Vivien et al. 1985; Bortone et al. 1989; Mapstone y Ayling 1998; Samoikys y Carlos 2000; Longo y Floeter 2012), existe una falta de consenso en relación a cuál es la técnica más apropiada y no ha sido posible establecer un estándar entre estas metodologías (Greene y Alevizon 1989). Por este motivo es conveniente realizar estas comparaciones metodológicas antes de comenzar un estudio a largo plazo en una determinada zona. De esta forma, nos aseguraremos que estamos trabajando con el método más adecuado.

Una de las principales razones del éxito de este tipo de metodologías visuales es el hecho de que permiten realizar estimaciones no destructivas de las comunidades de peces de zonas litorales (Cheal y Thompson 1997). Existen zonas en las que, por medidas de conservación, reglamentarias o debido a condiciones ambientales particulares, las técnicas no destructivas se convierten en la opción más acertada. Desde hace ya unas décadas existe un interés especial en la evaluación visual de las comunidades de peces en zonas tropicales (Harmelin-Vivien et al. 1985). En este contexto, los datos de una comunidad de peces de arrecifes coralinos, obtenidos mediante el uso de estas técnicas, tienen la ventaja de eliminar los efectos destructivos de las prácticas tradicionales tales como el dragado, el arrastre o el uso de anestésicos (Bardach 1959). Esto es muy importante en lugares donde no es conveniente perturbar el ambiente debido a las medidas de conservación del área en cuestión (Harmelin-Vivien et al. 1985), como es el caso de la Reserva Natural Marina de la Bahía de Murdeira donde se ha llevado a cabo este estudio. Al tratarse de un área marina protegida (AMP), ha de ponerse en práctica la metodología menos destructiva para evitar la perturbación de un ecosistema tan singular y, asimismo, dar ejemplo de buenas prácticas en un lugar en el que todavía no se ha afianzado la valoración y el respeto por la riqueza natural.

Una creciente preocupación por el equilibrio ecológico y económico entre la explotación y la conservación de los recursos naturales ha generado una mayor presión sobre los gestores ambientales y sus prácticas. Por lo que es común utilizar los datos procedentes de censos visuales como base para elaborar estrategias de manejo

* Departamento de Biología animal, Edafología y Geología. Universidad de La Laguna. Tenerife, Islas Canarias. España.

** Departamento de Engenharias e Ciências do Mar. Universidade de Cabo Verde. Mindelo, Cabo Verde.

*** Universidad de La Laguna. Corresponding author: jocarher@ull.es. 
y conservación (Buckley y Hueckel 1989). La información derivada de este tipo de muestreos es de carácter cuantitativa y está destinada a proporcionar un contexto empírico para la toma de decisiones sobre la actuación frente a diversos impactos, medidas de protección o cambios en el ambiente (Mapstone y Ayling 1998). Por ello, los muestreos de campo han de ser diseñados cuidadosamente (Craik 1981) y ofrecer los resultados más precisos posibles, ya que la calidad y la cantidad de información derivada de estos métodos es crucial para desarrollar gestiones ambientales adecuadas (Hendee et al. 1990). Por esta razón, en los últimos años, ha habido un empeño considerable en refinar la metodología de las técnicas de muestreo visuales con el objetivo de generar estimaciones lo más fiables posible (Bortone et al. 1989; Samoilys y Carlos 2000).

Como hemos nombrado anteriormente, los censos visuales representan la técnica más práctica para el seguimiento de poblaciones de peces tropicales de aguas someras o poco profundas. Los muestreos visuales realizados en estos ambientes ofrecen rápidas estimas de abundancias relativas, tallas y biomasa de peces (Samoilys y Carlos 2000). Sin embargo, una serie de dificultades hacen que el estudio de las comunidades ícticas de arrecife, por cualquier método general, sea complicado. Éstas derivan de la «complejidad y la gran cantidad de atributos inherentes a los hábitos de vida de las comunidades de peces tropicales» (Bortone et al. 1986). Algunas de estas características son la gran riqueza de especies, patrones de comportamiento dispares y distribuciones irregulares, entre otras, hechos que hacen complicado el determinar un método de muestreo estándar para su estudio (Samoilys y Carlos 2000). Además de estas dificultades, inherentes a las características de las poblaciones de peces, existen limitaciones logísticas en cada una de las técnicas visuales in situ desarrolladas hasta ahora. Las condiciones ambientales no favorables tales como la poca visibilidad, fuertes corrientes, sustratos muy irregulares u otros problemas relacionados con la viabilidad añaden dificultad a la implementación de algunos de estos métodos. Estos problemas ponen de manifiesto la necesidad de probar técnicas visuales específicas que permitan un estudio eficaz y preciso de las comunidades de peces presentes en ambientes diferentes (Bortone et al. 1989). Por otro lado, la precisión de las estimas al utilizar este tipo de técnicas puede estar influenciada por los sesgos introducidos por el propio observador, como la rapidez al nadar, la distancia a la cual se realiza el muestreo y la técnica de búsqueda. Si bien, estos últimos pueden ser reducidos considerablemente mediante un entrenamiento adecuado (Sale y Sharp 1983).

El método de muestreo visual elegido es determinante y las estimas de peces pueden variar con las dimensiones o medidas elegidas en cada método (Cheal y Thompson 1997). En la búsqueda del método más adecuado, Bortone et al. (1989) compararon el método "al azar» (Random), el de «Transecto» y el de "Punto fijo» bajo una amplia variedad de condiciones ambientales. La conclusión de su estudio fue que todos los métodos comparados eran equivalentes en cuanto a la capacidad de describir una comunidad de peces. En general, los métodos que ofrecían más información, puesto que sirven para asociar con mayor precisión los parámetros ambientales con una comunidad particular, eran aquellos que generaban menos varianza. Por otro lado, teniendo también en cuenta el hecho de que el buceo científico resulta 
caro y está limitado por la relación profundidad-tiempo, resulta fundamental desarrollar técnicas in situ que permitan optimizar y resolver este tipo de inconvenientes, siendo más apropiado aquel método que sea capaz de reunir la mayor cantidad de información en el menor tiempo posible, sin comprometer la fiabilidad de los resultados obtenidos (Bortone et al. 1989).

En términos de coste, sería deseable un tiempo de muestreo corto pero, si el número de peces es alto y la topografía compleja, el observador requerirá de un tiempo considerable para contabilizar debidamente. Por otro lado, el número de réplicas es un componente esencial en el diseńo experimental, si las réplicas son pocas el poder para detectar diferencias será muy bajo y si el número de réplicas es muy alto se traduce en una pérdida de esfuerzo (Bros y Cowell 1987). En definitiva, los métodos de muestreo elegidos deberían ser aquellos que ofrezcan datos fiables, maximicen el potencial para identificar cambios en las variables de la biota a estudiar, sean logísticamente factibles para ser utilizados en un amplio rango de situaciones y sean baratos; de manera que se pueda obtener la máxima flexibilidad en el diseño del muestreo dentro del presupuesto (Mapstone y Ayling 1998). Actualmente, el método de "Transecto" y el de "Punto fijo» son los métodos más utilizados por los investigadores que trabajan con comunidades de peces (Bohnsack y Bannerot 1986; Buckley y Hueckel 1989; Bortone et al. 1989,1991; Mapstone y Ayling 1998; Samoilys y Carlos 2000; Milazzo et al. 2011; Falcón 2015).

En relación a todos los argumentos anteriores, y con el afán de determinar cuál es el método más apropiado para el estudio de las comunidades ícticas en ambientes tropicales, este estudio tiene como objetivo principal evaluar y comparar la efectividad de dos métodos de muestreo visual subacuático: el de "Transecto» (T) y el de «Punto fijo» o "Circular» (C), en el arrecife coralino de la Reserva Natural Marina de la Bahía de Murdeira, con el fin de establecer qué método es el más fiable para llevar a cabo los seguimiento de las comunidades de peces en este espacio protegido.

\section{MATERIAL Y MÉTODOS}

\section{Área de ESTUdio}

Las islas de Cabo Verde se encuentran situadas en una franja que va desde 500 a $750 \mathrm{~km}$ al Oeste del continente africano, en el margen oriental del Océano Atlántico $\left(14^{\circ} 50^{\prime}-17^{\circ} 20^{\prime} \mathrm{N} ; 22^{\circ} 40^{\prime}-25^{\circ} 30^{\prime} \mathrm{W}\right)$. El archipiélago está conformado por diez islas y ocho islotes, formados por los acúmulos de roca de las erupciones causadas por un punto caliente bajo la plataforma submarina (Vinnik et al. 2012).

Anteriormente, según el concepto clásico empleado en biogeografía terrestre, el archipiélago de Cabo Verde era integrado, junto a Canarias, Madeira, Salvajes y Azores, en la región biogeográfica de la Macaronesia, perteneciente a la provincia cálido-templada Lusitana, siendo las islas más antiguas de esta ecoregión. La validez de esta agrupación como entidad biogeográfrica se encuentra cada vez más cuestionada tanto desde el punto de vista terrestre (Fernández-Palacios y Días 2001), 
como marino (Lloris et al. 1991; Falcón 2015). Desde el punto de vista marino, estudios recientes sobre ictiogeografía han contribuido a esclarecer el hecho de que las islas de Cabo Verde no pertenecen a dicha ecoregión marina de la Macaronesia, a la que pertenecen el resto de archipiélagos citados anteriormente, sino que forman parte de otra provincia, la llamada Atlántico Oriental Tropical (Briggs y Bowen 2012; Falcón 2015).

En relación a las influencias bajo las que está sometido el archipiélago, con respecto a la circulación oceánica, Cabo Verde se encuentra en el límite sur de la Corriente de Canarias, la cual sufre una desviación en la costa oriental africana hacia el Oeste y, gradualmente, se convierte en la Corriente Ecuatorial del Norte. Por otro lado, a latitudes más bajas, se encuentra la Contracorriente Nor-Ecuatorial o Corriente Ecuatorial del Sur con flujo en dirección Este (Fernandes et al. 2004). En este contexto, bajo la influencia de la Corriente de Canarias o su derivación y la Corriente Nor-Ecuatorial, las islas presentan una estación fría de diciembre a junio caracterizada por la llegada de aguas frías y ricas en nutrientes y, por otro lado, de julio a noviembre, las islas se encuentran bajo la influencia de la Contracorriente Nor-Ecuatorial con presencia de aguas más cálidas (Almeida et al. 2007).

En relación a la comunidad íctica del archipiélago, la ictiofauna de Cabo Verde está representada por un número considerable de especies, siendo reconocidas 315 especies hasta el año 2013 (Wirtz et al. 2013). En comparación con los archipiélagos de la Macaronesia (Canarias, Madeira, Salvajes y Azores), la composición zoogeográfica de la ictiofauna costera de Cabo Verde es en su mayor parte tropical, con una clara dominancia de especies guineanas, seguida de especies anfiatlánticas tropicales y subtropicales, aunque también con presencia de especies de amplia distribución en el Atlántico Oriental cálido-templado, del Mediterráneo y circuntropicales (Brito et al. 1999, 2007, 2011; Falcón 2015). En contraste con los archipiélagos macaronésicos, Cabo Verde es el que presenta mayor biodiversidad y endemicidad del Atlántico (Brito et al. 2007, 2011; Freitas 2014; Falcón 2015).

Este estudio se llevó a cabo en la Reserva Natural Marina de la Bahía de Murdeira situada al suroeste de la isla de Sal (16³3,5’N; 22055,6’W), Cabo Verde (figura 1). Esta bahía fue declarada Reserva Natural Marina en la Ley de Espacios Naturales Protegidos de Cabo Verde (Decreto-Lei n³/2003, de 24 de Fevereiro) por su gran riqueza e importancia de los ecosistemas submarinos, presentando una elevada proporción de endemismos y singularidades, así como por la presencia de recursos pesqueros y costeros (Almeida 2013). El litoral de esta bahía se compone principalmente de depósitos sedimentarios del Cuaternario con una anchura de cerca de $1 \mathrm{~km}$, a excepción del promontorio basáltico al norte, denominado Monte Rabo de Junco (Torres et al. 2002). La bahía mantiene casi todo el año unas condiciones de aguas calmadas que permiten una estratificación térmica muy estable a lo largo del tiempo, además de fondos rasos que no suelen superar los 10 metros de profundidad en la parte interna (Almeida et al. 2007). En la zona más abrigada de la región infralitoral, especialmente en Calheta Funda, Jorge Fonseca o próximas a la punta de Rabo de Junco, se encuentran colonias de corales más o menos extensos de las especies: Millepora alcicornis, Porites astreoides, Siderastrea radians, Favia fragum y Palythoa caribbaeorum (Lopez-Jurado 1998; Almeida et al. 2007). 
En general, los corales hermatípicos y los sustratos con S. radians son muy comunes en los fondos rocosos poco profundos de las islas del Archipiélago (Morri et al. 2000; Freitas 2014). Debido a la influencia de la Corriente fría de Canarias, la temperatura del agua no permite las formaciones coralinas extensas y dominantes, por lo que los arrecifes de coral en Cabo Verde también están compuestos en gran medida por esponjas y algas, que crecen sobre sustratos duros formados por restos de coral muerto, algas calcáreas y otros organismos depositados sobre material volcánico (Van der Land 1993; Almeida et al. 2007; Freitas 2014). Por esta razón, es necesario puntualizar que, aunque en este estudio se hace referencia a arrecifes de coral, en el caso de Cabo Verde este término está representado por comunidades coralinas basadas en un complejo ambiente formado por depósitos de carbonatos y corales junto con la biota asociada (Almeida et al. 2007; Freitas 2014).

Para llevar a cabo el estudio se seleccionaron, en el interior de la bahía, 8 localidades al azar $\left(1=16^{\circ} 67,9^{\prime} \mathrm{N} 022^{\circ} 96,2^{\prime} \mathrm{W} ; 2=16^{\circ} 66,3^{\prime} \mathrm{N} 022^{\circ} 94,6^{\prime} \mathrm{W} ; 3=\right.$ $16^{\circ} 66,1^{\prime} \mathrm{N} 022^{\circ} 95,9^{\prime} \mathrm{W} ; 4=16^{\circ} 66,7^{\prime} \mathrm{N} 022^{\circ} 95,16^{\prime} \mathrm{W} ; 5=16^{\circ} 68,0^{\prime} \mathrm{N} 022^{\circ} 95,1^{\prime} \mathrm{W}$; $\left.6=16^{\circ} 68,7^{\prime} \mathrm{N} 022^{\circ} 97,7^{\prime} \mathrm{W} ; 7=16^{\circ} 69,4^{\prime} \mathrm{N} 022^{\circ} 97,3^{\prime} \mathrm{W} ; 8=16^{\circ} 69,5^{\prime} \mathrm{N} 022^{\circ} 95,3^{\prime} \mathrm{W}\right)$ (figura 1). Estas localidades estaban repartidas por toda la bahía, para abarcar todos los posibles ambientes. Éstas presentaban diferentes estructuras ambientales en relación a la cobertura de coral, porcentaje de arena, inclinación y complejidad topográfica. Las especies de peces fueron registradas en un rango de profundidad de entre 3 y $18 \mathrm{~m}$ mediante censos visuales con el método de «Transecto» (T) y con el método de «Punto fijo o Circular» (C) (figura 2).

\section{EsPeCIES}

Durante los muestreos se anotaron las abundancias y tallas de todas las especies que aparecían en uno y otro método de muestreo. Todas las especies contadas fueron registradas por su nombre científico y agrupadas por familias, destacando además aquellas especies endémicas del Archipiélago, según la información recogida en Fishbase (Froese y Pauly 2015) y en trabajos como en el de Smith et al. (2007), Fernández-Gil et al. (2013), Wirtz et al. (2013) y Falcón (2015) (tabla 1). Además, se elaboró un catálogo fotográfico con todas las especies muestreadas que será de gran valor para los futuros seguimientos de la Reserva (Anexo 1).

\section{Métodos de muestreo subacuÁtico}

Los métodos visuales in situ han demostrado ser, de todas las técnicas utilizadas, los menos perjudiciales para el recuento de peces litorales en aguas someras (Bardach 1959; Mapstone y Ayling 1998). En este estudio, los muestreos de las comunidades de peces se realizaron in situ con la utilización de escafandra autónoma por parte de los observadores, haciendo uso de dos métodos de recuento visual subacuático: «Transecto» (T) y «Punto fijo/Circular» (C) (figura 2). Para minimizar los errores y posibles sesgos, los procedimientos en la práctica de los censos visuales con 
ambos métodos se estandarizaron en la medida de lo posible, mediante la división de los recuentos entre dos observadores debidamente entrenados (Samoilys y Carlos 2000). Para eliminar la perturbación debida al fondeo de la embarcación y a la entrada de los buceadores en el agua, los dos observadores se alejaron cierta distancia antes de comenzar el recuento. Para intentar solventar los inconvenientes debido a las posibles variantes dentro de cada método (velocidad de aleteo, dimensiones del transecto y radio del punto fijo), en el presente estudio los recuentos fueron realizados a lo largo del transecto a una velocidad tal que no fuera ni demasiado rápida para no subestimar especies ni demasiado lenta para evitar contar más de una vez los mismos individuos; y se estableció, en el método circular un radio de 5,6m que permitía distinguir y registrar la mayoría de peces de mayor o menor tamaño y de más o menos movilidad. Los muestreos se realizaron de mayor a menor profundidad para evitar problemas asociados al buceo.

Durante los muestreos, los datos eran apuntados a lápiz sobre tablillas de PVC en papel resistente al agua donde se registraban todas las especies presentes, es decir, en cada muestreo se anotaron todas las especies que aparecieron dentro del área de cada método. Aun siendo difícil para el observador el tratar de estimar visualmente un amplio rango de tallas de peces, especialmente peces pequeños (Bellwood y Alcala 1988), se tuvieron en cuenta, como se cita anteriormente, todas las especies presentes de cualquier tamaño. Además de registrar los datos referentes a la comunidad de peces, se anotaron algunas características del entorno en cada punto de muestreo, como: fecha, hora, tipo de sustrato, porcentaje de área cubierto por arena y profundidad.

\section{Transecto (T)}

El método de transecto es una técnica ampliamente utilizada (Krajewski y Floeter 2011) que permite el recuento de todos los peces móviles de mayor tamaño y mejora el registro de las especies más crípticas, lo cual permite obtener una buena estimación de densidad para todas las especies (Ferreira et al. 2001, 2004). En cada localidad se llevó a cabo un determinado número de transectos, de 3 a 6 réplicas, y la posición de estos fue escogida al azar dentro de la Bahía (Freitas 2012). Se utilizaron transectos de $20 \times 2 \mathrm{~m}\left(40 \mathrm{~m}^{2}\right)$, de manera que el buceador realizaba un recorrido de ida desde un extremo del transecto, registrando todas las especies presentes en un ancho de $1 \mathrm{~m}$ desde la línea del transecto hacia la derecha y repitiendo este procedimiento en el camino de vuelta (figura $2 \mathrm{a}$ ).

\section{Punto fijo o Circular (C)}

Esta técnica de muestreo, descrita previamente por Bortone et al. (1989) y modificada posteriormente por Falcón et al. (1993), se basa en un observador provisto de un equipo de submarinismo, situado en un punto al azar dentro de la localidad de estudio. Este punto simboliza el punto central de un cilindro imaginario 
de 5,6 $\mathrm{m}$ de radio, que se extiende desde la superficie hasta el fondo, representando una superficie de $100 \mathrm{~m}^{2}$ en total, siendo esta medida útil para el muestreo de un amplio rango de peces de arrecife incluido las especies de tamaños más pequeños (Bortone et al. 1991). El radio de la circunferencia es marcado con una cuerda, en cuyo extremo se situaba el observador para comenzar el recuento, tomando como referencia el extremo opuesto de la cuerda como límite del área de muestreo (figura 2b). A continuación, el observador comienza a girar sobre sí mismo, llevando a cabo el registro de todas las especies observadas. Con el fin de reducir sesgos debidos a la detección diferencial de especies, el muestreo es realizado de forma estratificada, es decir, durante los primeros cinco minutos se le da prioridad a aquellas especies más móviles que pudieran abandonar rápido la zona de muestreo y posteriormente, se procedía al recuento de las especies crípticas. Siguiendo el criterio de Brock (1954), todos los peces que entran dentro del área del cilindro imaginario son contadas de manera que si parte de un cardumen de peces entra dentro de este área, todos los peces del cardumen son registrados. En el caso de recuentos de cardúmenes grandes de peces, se contaban los individuos de una porción y, a partir de ahí, se estimaba el conjunto completo. Los peces que entraran por segunda vez en el área de muestreo no son registrados.

\section{ANÁlisis DE DATOS}

En primer lugar, se llevó a cabo una recopilación, a modo de análisis descriptivo, de las familias y las respectivas especies registradas en los muestreos, representando el número total de familias y especies así como la abundancia obtenida para cada una de ellas (tabla 1). A continuación, con el objetivo de estudiar la posible influencia del tipo de muestreo utilizado ("Transecto» o "Punto fijo») sobre la riqueza y la abundancia de especies, se llevó a cabo un análisis multivariante de la varianza (PERMANOVA) por permutaciones (Anderson et al. 2001). Para el análisis se utilizó un diseño de dos vías considerando, en primer lugar, el factor «Método», siendo fijo y con dos niveles (1: «Transecto", 2: "Circular») y, en segundo lugar, el factor «Localidad», aleatorio y con ocho niveles $(1,2,3,4,5,6,7,8)$. Por otro lado, la influencia de dichos factores se estudió sobre las variables riqueza, referida al número de diferentes especies presentes, y abundancia, que hace referencia al número de individuos de cada especie.

En el caso de la riqueza íctica, los datos de abundancias fueron transformados con Presencia/Ausencia. Los datos de abundancias fueron transformados con $\log (\mathrm{X}+1)$. En ambos casos las matrices triangulares se obtuvieron aplicando la disimilaridad de Bray-Curtis, que ha demostrado ser una robusta herramienta en el tratamiento de muestras biológicas (Bray y Curtis 1957). Además de realizar el análisis PERMANOVA para las dos variables a estudiar, riqueza íctica y abundancia, se llevó a cabo el análisis de comparaciones "pairwise» (por pares) a posteriori entre el método de muestreo y cada una de las distintas localidades para poner de manifiesto las posibles diferencias en las estimas de riqueza y abundancia íctica entre métodos de muestreo y localidades. Para conocer qué especies marcaban la disi- 
milaridad entre localidades y entre los métodos de muestreo para la localidad 2, se realizó un análisis SIMPER (Similarity Percentage Analysis) (Clarke 1993; Clarke y Warwick 1994). Posteriormente, se seleccionaron aquellas especies que contribuían a explicar el 60\% de la variación y que marcaron las diferencias entre localidades y entre métodos.

Además, para visualizar mejor los resultados obtenidos, se realizaron análisis de ordenación de componentes principales (PCO) para cada una de las variables, riqueza y abundancia íctica (figuras 3 y 4). En primer lugar se llevó a cabo un PCO general incluyendo todos los muestreos y posteriormente se realizó otro PCO parcial para la «Localidad 2». Además, para cada variable graficada se superpusieron, mediante ejes vectoriales, los resultados obtenidos en el análisis SIMPER, utilizando únicamente las especies que contribuyeron hasta un $60 \%$ a la diferenciación entre localidades y entre métodos en el caso de la localidad 2.

Asimismo, para la variable riqueza íctica, se llevó a cabo otro análisis más exhaustivo para conocer qué especies fueron registradas sólo por un método de muestreo y no por el otro. Para ello se realizó, en primer lugar, un análisis de la Riqueza (S) y por último, se evaluó la Diversidad existente en cada una de las ocho localidades, calculada mediante el índice de Margalef $[\mathrm{d}=(\mathrm{S}-1) / \mathrm{lnN}]$; donde $\mathrm{S}$ es el número de especies y $\mathrm{N}$ el número total de individuos. Con este índice, localidades con valores inferiores a 2 son consideradas como zonas de baja biodiversidad mientras que localidades con valores superiores a 5 son indicativos de zonas de alta biodiversidad (Margalef 1956). Por último, se representó gráficamente la relación entre el nivel de significación, obtenido en el análisis a posteriori de la variable abundancia y el índice de Margalef resultante para cada localidad. Este último análisis pretende comprobar la relación entre la diversidad de una localidad y las diferencias obtenidas entre los métodos de muestreo.

Para realizar todos estos análisis, los datos fueron tratados con el paquete estadístico PRIMER 6 \& PERMANOVA+ v.1.0.1. En los análisis de varianza realizados para este estudio se utilizaron 4999 permutaciones, estableciéndose un nivel de significación de $\alpha=0,05$.

\section{RESULTADOS}

\section{ANÁLISIS DESCRIPTIVO}

Durante los muestreos realizados en la Bahía se registraron un total de 5018 individuos repartidos en 24 familias, 41 géneros y 50 especies (tabla 1; Anexo 1). La familia con más representantes fue Pomacentridae seguida de Sparidae. Entre las especies más abundantes se encuentran, de mayor a menor abundancia: Chromis lubbocki, Chromis multilineata, Parapristipoma humile, Gobius tetrophthalmus y Stegastes imbricatus. Por otro lado, entre las menos abundantes, sólo con un individuo registrado, encontramos: Similiparma lurida, Chilomycterus reticulatus, Epinephelus costae, Labrisomus nuchipinnis y Ophioblennius atlanticus. Entre las especies registradas, destacan los siguientes 10 endemismos (Fernández-Gil et al. 2013; Wirtz et al. 2013; Falcón 2015): Parablennius salensis, Gobius ateriformis, Gobius tetrophthalmus, 
Parapristipoma humile, Chromis lubbocki, Similiparma hermani, Diplodus fasciatus, Diplodus sargus lineatus, Diplodus prayensis y Virididentex acromegalus.

\section{RiqueZA DE ESPECIES}

El análisis PERMANOVA en relación con la presencia/ausencia de especies como medida de riqueza, mostró que no existen diferencias significativas en las estimas en relación al "Método». Sin embargo, existen diferencias significativas en la interacción de los factores «MétodoxLocalidad» y en el factor «Localidad» pero, en este último caso, al tratarse de un factor aleatorio no tiene un test de hipótesis asociado por lo que en este estudio no analizaremos en detalle los efectos de la localidad, sino que se valorará el resultado del análisis con respecto al factor fijo «Método» y la interacción de los dos factores «MétodoxLocalidad» (tabla 2a). En el análisis a posteriori, la única localidad que presenta diferencias en las estimas, según el método de muestreo utilizado, es la Localidad 2, no habiendo diferencias significativas en los registros de las siete localidades restantes entre métodos (tabla 2b).

El análisis SIMPER realizado para las ocho localidades mostró que las especies que más contribuyen a diferenciar dichas localidades entre sí son: Acanthurus monroviae, Cephalopholis taeniops, Chromis lubbocki, Chromis multilineata, Gnatholepis thompsoni, Gobius tetrophthalmus, Parablennius salensis, Pseudupeneus prayensis, Stegastes imbricatus, Sargocentron hastatum y Thalassoma pavo (tabla 3). Asimismo, el análisis SIMPER únicamente para la localidad 2, que mostraba una disimilaridad del 63,84\% en las estimas según el método de muestreo empleado, determinó que las nueve especies que contribuyen a marcar dicha diferencia entre métodos son: Abudefduf hoefleri, Balistes punctatus, Canthigaster capistrata, Chromis multilineata, Coris atlantica, Thalassoma pavo, Sargocentron hastatum, Scarus hoefleri y Sparisoma cretense, es decir, estas especies fueron muestreadas por uno de los dos métodos y no por el otro (tabla 4).

En el análisis PCO para la variable riqueza íctica se observa una dispersión homogénea de los muestreos sin ninguna separación por métodos de muestreo (figura 3a). En el caso de las localidades aparece un cierto grado de agrupamiento en alguna de las localidades y se observa separación entre las mismas. Esta separación entre localidades, además, parece ser debida a la presencia de especies con distintas apetencias de hábitat, hacia la derecha del gráfico aparecen especies características de ambientes más arenosos y hacia la izquierda, especies de arrecife coralino (figura 3b). La ordenación PCO parcial, realizada para la Localidad 2, explica el $62 \%$ de la variabilidad de los datos y se puede observar claramente qué especies caracterizaron a uno u otro método. Por ejemplo, para el método T éstas fueron Sparisoma cretense y Thalassoma pavo mientras que, por otro lado, Abudefduf hoefleri, Balistes punctatus, Canthigaster capistrata, Chromis multilineata, Coris atlantica y Sargocentron hastatum fueron registradas por el método circular (figura 3c).

En relación al análisis más exhaustivo, realizado para poner de manifiesto aquellas especies que fueron únicamente registradas por uno de los métodos, tomando como referencia la riqueza (S), se observa una diferencia de cinco especies entre el 
método $\mathrm{T}$ y el método circular. Las especies registradas por el muestreo de tipo $\mathrm{T}$ y no por el C fueron Diplodus puntazzo, Gobius ateriformis, Labrisomus nuchipinnis, Scorpaena maderensis y Sphoeroides marmoratus. Por otro lado, las especies contabilizadas por el muestreo C y no por el T fueron Caranx crysos, Chilomycterus reticulatus, Diodon holocanthus, Epinephelus costae, Ophioblennius atlanticus, Priacanthus arenatus, Scorpaena laevis, Similiparma lurida, Synodus synodus y Virididentex acromegalus. Además, existe una diferencia en el total de individuos registrados $(\mathrm{N})$, el método circular muestreó 12 individuos más que el método de transecto (tabla 5).

\section{Abundancia}

El análisis de PERMANOVA para evaluar la variación con respecto a la abundancia de especies, encontró los mismos resultados que en el análisis de la riqueza íctica. Es decir, que ambos métodos estiman por igual la abundancia de especies pero existen diferencias significativas en la interacción de los factores «MétodoxLocalidad» (tabla 6a). Para conocer la localidad concreta en la que existen diferencias en las estimas de abundancia según el método utilizado se realizaron las comparaciones por pares a posteriori indicando. En este caso también, resultó ser la localidad 2 aquella en la que existen diferencias en las abundancias registradas según el método de muestreo utilizado (tabla 6b).

En el análisis SIMPER de las ocho localidades para la variable abundancia se obtuvieron los mismos resultados que para la riqueza íctica (tabla 7). Por otro lado, el análisis SIMPER parcial de la Localidad 2, estableció que las ocho especies que más contribuyen a marcar la diferencia entre métodos son: Aulostomus strigosus, Chromis multilineata, Pseudupeneus prayensis, Scarus hoefleri, Sparisoma cretense, Coris atlantica, Stegastes imbricatus y Thalassoma pavo, con un porcentaje de disimilaridad entre métodos del 62,48\% (tabla 8).

En relación a la abundancia, la ordenación PCO muestra una dispersión homogénea de los muestreos sin separación debidas al método de muestreo (figura 4a). En el caso de las localidades, aparece cierta segregación entre las mismas. La superposición de las especies seleccionadas previo análisis SIMPER aclara que la separación entre las localidades se debe principalmente a las características del hábitat muestreado, zonas más arenosas hacia la derecha del gráfico y coralinas hacia la izquierda (figura 4b). El análisis de componentes principales parcial realizado para la Localidad 2, que explica un 59,4\% de la variación de los datos, resalta las diferencias entre los dos métodos de muestreo utilizado, apareciendo nuevamente superpuestas las especies que, según el análisis SIMPER, marcaron la diferencia entre uno y otro método (figura $4 \mathrm{c}$ ). 
En el análisis de la diversidad utilizando el índice de Margalef como referencia, se obtuvo que la Localidad 2 resultó ser la más diversa (tabla 9), con un valor de 7,145 y la localidad 8 la menos diversa con un valor de 2,682. En la figura 5 se correlaciona la diversidad que presenta cada localidad y el p-valor obtenido en las comparaciones por pares de la abundancia de especies entre métodos. Es decir, se relaciona el grado de diferencia entre métodos de muestreo con la diversidad del lugar. Se puede observar que, cuanto más diversa es la localidad menor es el p-valor y, por tanto, más marcada la diferencia en las estimas de cada método de muestreo para esa localidad concreta (figura 5).

\section{DISCUSIÓN}

Los resultados obtenidos en este estudio, para la riqueza y la abundancia íctica, indican de manera similar al estudio llevado a cabo por Samoilys y Carlos (2000) en zonas de arrecife, que en general, las dos técnicas de muestreo utilizadas resultan igualmente efectivas y, por lo tanto, ambas son válidas a la hora de realizar muestreos de peces en Cabo Verde. Sin embargo, la obtención de diferencias significativas en la interacción del factor «MétodoxLocalidad» se traduce en que las estimas de riqueza y abundancia de especies difieren con la utilización de un método u otro según la localidad. Los resultados del análisis a posteriori revelaron diferencias en las estimas de los dos tipos de muestreo únicamente en la localidad 2, que resultó ser la localidad con más diversidad de todas. Por lo que hay que tener especial precaución en los muestreos que se llevan a cabo en localidades con mayor diversidad.

A pesar de que, en general, los dos métodos son igual de efectivos a la hora de registrar la riqueza y la abundancia de especies, el estudio pormenorizado de las especies que aparecen en uno u otro método sugiere que el método circular es capaz de registrar mayor número de especies de peces e individuos que el método de transecto. En este sentido, Harmelin-Vivien et al. (1985) señalan que el método circular es especialmente apropiado en zonas de fondos rocosos muy heterogéneos, como es el caso de nuestra Localidad 2, que presenta gran complejidad coralina y diversidad íctica. Por estas razones, y además debido a que el método circular podría ser llevado a cabo en el 70\% del tiempo utilizado para muestrear sobre un transecto (Samoilys y Carlos 2000), resulta lógico considerar que el método circular es el más eficiente y el que debería utilizarse en los seguimientos de la Reserva Natural de la Bahía de Murdeira.

A pesar de que la Bahía de Murdeira no haya sido muy estudiada y todavía se requieran más investigaciones, la ictiofauna de Cabo Verde sí ha sido estudiada desde hace ya algún tiempo y cada vez salen a la luz más aportaciones (Brito et al. 1999; Wirtz et al. 2013; Freitas, 2014; Falcón 2015). Basándonos en un estudio descriptivo reciente sobre peces costeros de Cabo Verde, se registró un total de 315 especies de peces, $88 \%$ de las cuales resultaron ser teleósteos (Wirtz et al. 2013; Freitas 2014). De las 278 especies de peces teleósteos, un total de 50 especies fue- 
ron registradas durante los muestreos del presente estudio. En el caso de las especies endémicas, de las 20 especies presentes en el Archipiélago (Fernández-Gil et al. 2013; Wirtz et al. 2013; Freitas 2014), 10 fueron encontradas durante el desarrollo de las campañas de muestreo (tabla 1). Por lo que el presente trabajo resulta fundamental dado que la zona de estudio es un área con gran número de endemismos y la utilización de métodos de muestreo no destructivos es fundamental en este tipo de situaciones.

La variedad de especies que hemos encontrado durante nuestro estudio pone de manifiesto también los diferentes ambientes muestreados. En ambientes donde predomina la arena, aparecen especies territoriales, más crípticas o sedentarias tales como los góbidos o blénidos. Por otro lado, en ambientes rocosos o intersección son comunes especies de mayor movilidad y tamaño como por ejemplo las pertenecientes a las familias Scaridae, Sparidae, Mullidae, etc. En ambientes de paredes, veriles, grietas o cuevas aparecen especies de hábitos más gregarios, formando grupos, como las de las familias Pomacentridae o Apogonidae (Almeida et al. 2007). Basándonos en esto, podemos decir que las localidades 1, 3, 5 y 6 están representadas por ambientes de tipo arenoso o de transición arena-roca, caracterizados por la presencia de especies típicas de este tipo de fondos como Gobius tetrophthalmus, Parablennius salensis, Gnatholepis thompsoni, Pseudupeneus prayensis y Cephalopholis taeniops. En el caso de las localidades 2, 4, 7 y 8, están caracterizadas por ambientes más complejos de roca y coral, con presencia de oquedades y grietas, marcadas por la presencia de especies como Chromis lubbocki, Chromis multilineata, Thalassoma pavo, Acanthurus monroviae, Sargocentron hastatum y Stegastes imbricatus (Froese y Pauly 2015).

Es conveniente señalar que la mayoría de los estudios que han empleado métodos de muestreo visual para estimar la riqueza y abundancia de peces de un lugar, no suelen contar con extensos estudios piloto previos, como el presente trabajo. En esos casos la elección del mejor método normalmente está determinada por la experiencia del investigador, sentido común, antecedentes sobre los métodos usados previamente, etc. (Samoilys y Carlos 2000). Sin embargo, es fundamental realizar este tipo de comparaciones en zonas que no han sido muy estudiadas o en las cuales se pretende llevar a cabo un seguimiento a largo plazo, a fin de determinar el método más adecuado y optimizar así el trabajo de campo posterior.

Existen ciertas limitaciones o inconvenientes al utilizar este tipo de métodos que pueden influir en los resultados. A pesar de que se ha demostrado el potencial de los métodos visuales subacuáticos para estimar la diversidad de peces en general (Watson et al. 1995), éstos suelen subestimar los valores de riqueza y abundancia (Brock 1982; García-Charton et al. 2000). En general, se suelen subestimar las especies más crípticas y las más abundantes por su dificultad para ser localizadas o contadas respectivamente. Otro punto crítico puede ser el uso de dos observadores distintos que, a pesar de estar entrenados, pueden interpretar de manera diferente el ambiente, obteniendo registros diferentes durante los muestreos. En nuestro caso, los muestreadores poseían amplia experiencia y sólo se detectó efecto del método en la localidad 2. Para este tipo de métodos la precisión de los resultados obtenidos depende en gran medida de la pericia del investigador, así como de su conocimiento sobre el comportamiento de las especies durante el censo (Brock 1982). De ahí la 
importancia de someter a los observadores a re-calibraciones periódicas, cuando son varios los que realizarán el seguimiento, para asegurar que los sesgos producidos por estos se mantengan constantes (Mapstone y Ayling 1998).

Centrándonos en cada uno de los métodos, la técnica de transecto es un método simple al mismo tiempo que permite a los observadores concentrarse en el área que tienen delante de ellos, de manera que se reducen las complicaciones como resultado de intentar ver los peces que se encuentran en el límite de su alcance visual, como ocurriría en el método circular (Bortone et al. 1989). Además, se ha demostrado que con la técnica de transecto es más probable la subestimación de individuos que la sobreestima ya que, según Harmelin-Vivien et al. (1985), los observadores son más propensos a pasar por alto individuos que a contarlos dos veces. En relación a la técnica circular, ésta ha sido adaptada recientemente de los estudios de fauna terrestre (Bohnsack y Bannerot 1986). Ésta tiene la ventaja de no necesitar la colocación previa de la línea de referencia del transecto, lo cual supone un ahorro de esfuerzo y en consecuencia de consumo de aire bajo el agua, así como una menor perturbación del ambiente y de los peces de la zona antes de comenzar el recuento. Si bien, el muestreador necesita cierto entreno previo para estimar correctamente las distancias. Por otro lado, aparte de las ventajas y desventajas de los dos métodos en general, existen otros factores a tener en cuenta dentro de cada una de las técnicas y que pueden influir en las estimas realizadas, como por ejemplo, la velocidad de aleteo a lo largo del transecto, sus dimensiones, así como el tamaño del radio en el circular (Mapstone y Ayling 1993; Cheal y Thompson 1997). Durante el presente estudio todo este tipo de cuestiones intentaron solventarse adoptando una velocidad de aleteo y un radio de muestreo apropiados según la experiencia de los observadores y los estudios realizados anteriormente, de manera que no se comprometiera la fiabilidad de las estimas realizadas.

Estos métodos son, además, relativamente sencillos de aplicar, no precisan de trabajo posterior en el laboratorio, son económicos, permiten la obtención de numerosas variables, no alteran las poblaciones estudiadas y pueden utilizarse en cualquier tipo de hábitat, incluso en aquellos más delicados dentro de áreas marinas protegidas (Forcada 2007). En definitiva, con este trabajo hemos pretendido aportar información relevante a la hora de elegir el método a utilizar en futuros estudios de seguimiento en el Área Protegida de la Bahía de Murdeira, Cabo Verde.

\section{AGRADECIMIENTOS}

Queremos agradecer a la escuela de doctorado y a la comisión académica del máster de Biología Marina: Biodiversidad y Conservación de la Universidad de La Laguna el haber hecho posible el viaje de fin de máster a la isla de Sal en Cabo Verde durante el curso académico 2014/2015. 


\section{AUTHORS CONTRIBUTION}

Conceptualización: JCH

Metodología y trabajo en campo: RF, JCH, IS

Análisis de datos: JCH

Preparación del escrito original: IS, JCH

Corrección y edición del escrito definitivo: JCH, RF

RECiBIDO: enero de 2020; ACEPTADO: febrero de 2020 


\section{REFERENCIAS}

Almeida, C., Fernández, G.L., Elso, M.Z., Da Rosa, M.R., Jiménez, N.G., Sinter, P.M., Quintana, R.D. y Freitas, R.M. 2007. Availaçao do ambiente e recursos marinos da Baía da Murdeira, Sal - Cabo Verde. Projecto de Conservaçao Marinha e Costeira/WWF. Direcçao Geral do Ambiente, Governo de Cabo Verde. 139 p.

Almeida, C. 2013. Valoración ecológica del Área Marina Protegida de la Bahía de Murdeira, Isla de Sal, Cabo Verde. (Tesis Doctoral). Universidad de Las Palmas de Gran Canaria, España. $232 \mathrm{p}$.

Anderson, M.J. 2001. Permutation tests for univariate or multivariate analysis of variance and regression. Can. J. Fish. Aquat. 58: 626-639.

BARDACH, J.E. 1959. The summer standing crop of fish on a shallow Bermuda reef. Limnol. Oceanogr. 4: 77-85.

BeLlwood, D.R. 1988. On the use of visual survey methods for estimating reef fish standing stocks. Fishbyte. 6: 14-15.

Bellwood, D.R. y Alcala, A.C. 1988. The effect of a minimum length specification on visual estimates of density and biomass of coral reef fishes. Coral Reefs. 7: 23-27.

Bohnsack, J.A. y BAnNerot, S.P. 1986. A stationary visual census technique for quantitatively assessing community structure of coral reef fishes. NOAA Technical Report NMFS. 41: 1-15.

Bortone, S.A., Kimmel, J.J. y Bundrick, C.M. 1989. A comparison of three methods for visually assessing reef fish communities: time and area compensated. Northeast GulfSci. 10(2): 85-96.

Bortone, S.A., Van Tassel, J., Brito, A., Falcón, J.M. y Bundrick, C.M. 1991. A visual assessment of the inshore fishes and fishery resources off El Hierro, Canary Islands: A baseline survey. Sci. Mar. 55(3): 529-541.

Bray, J.R. y Curtis J.T. (1957): An ordination of the upland forest communities of Southern Wisconsin. Ecol. Monogr. 27: 325-349.

BRIGgs, J.C. y Bowen, B.W. 2012. A realignment of marine biogeographic provinces with particular reference to fih distributions. J. Biogeogr. 39: 12-30.

Brito, A., Falcón, J.M. y Herrera, R. 2007. Características zoogeográficas de la ictiofauna litoral de las islas de Cabo Verde y comparación con los archipiélagos macaronésicos. Rev. Acad. Canar. Cienc. 18: 93-109.

Brito, A., Freitas, R., Espino, F., Fernández-Gil, C., Boyra, A. y González, J.A. 2013. Fishes. En: Fernández-Gil, C., González, J.A. y González, N. (Eds). Espécies Marinhas de Cabo Verde. Biotecmar. pp. 32-73.

Brito, A., Herrera, R., Falcón, J.M., García-Charton, J.A., Barquín, J. y Pérez-Ruzafa, A. 1999. Contribución al conocimiento de la ictiofauna de las islas de Cabo Verde. Rev. Acad. Canar. Cienc. XI: 27-41.

Brock, R.E. 1982. A critique of the visual census method for assessing coral reef fish populations. Bull. Mar. Scienc. 32(1): 269-276.

Brock, V.E., 1954. A preliminary report on a method of estimating reef fish populations. J. Wildlife Manage. 18: 297-308.

Bros, W.E. y Cowell, B.C. 1987. A technique for optimizing sample size (replication). J. Exp. Mar. Biol. Ecol. 1: 63-71. 
Buckley, R.M. y Hueckel, G.J. 1989. Analysis of visual transects for fish assessment on artificial reefs. Bull. Mar. Scienc. 44: 893-898.

Cheal, A.J. y Thompson, A.A. 1997. Comparing visual counts of coral reef fish: Implications of transect with and species selection. Mar. Ecol. Prog. Ser. 158: 241-248.

Clarke, K. 1993. Non-parametric multivariate analyses of changes in community structure. Aust. J. Ecol. 18: 117-143.

Clarke, K. y Warwick, R. 1994. Change in marine communities: An approach to statistical analysis and interpretation. NERC-BAS. Plymouth, Reino Unido. $144 \mathrm{p}$.

Craik, G.J.S. 1981. Underwater survey of coral trout Plectropomus leopardus (Serranidae) populations in the Capricornia section of the Great Barrier Reef Park. Proceedings of the $4^{\text {th }}$ International Reef Symposium. 1: 53-58.

Duarte, M.C. y Romeiras, M.M. 2009. Cape Verde Islands. En: Gillespie, R.G. \& Clague, D.A. (Eds). Encyclopedia of Islands. University of California Press, Berkeley, United States. p. 143-150.

Fernandes, M.J., Lazaro, C., Santos, A.M.P. y Oliveira, P. 2004. Oceanographic characterization of the Cape Verde region using multisensor data. Proceedings of the 2004 Envisat \& ERC Symposium, Salzburg, Austria.

Fernández-Palacios, J.M. y Días, E. 2001. Marco biogeográfico macaronésico, en Fernández-Palacios, J.M. \& Martín Esquivel, J.L. (eds.). Naturaleza de las Islas Canarias. Ecología y Conservación. Editorial Turquesa. Santa Cruz de Tenerife, España. p. 45-52.

Ferreira, C.E.L., Goncalves, J.E.A. y Coutinho, R. 2001. Community structure of fishes and habitat complexity on a tropical rocky shore. Environ. Biol. Fish. 61: 353-369.

ForCadA, A. 2007. Evaluación de las areas marinas protegidas y su efecto en pesquerías artesanales del Mediterráneo Occidental. Tesis doctoral. Universidad de Alicante, España.

Freitas, R.M. 2012. Reef fish and benthic community structure of Santa Luzia marine reserve, Eastern Atlantic. Trabajo post-graduación. Universidad de Cabo Verde y Universidad del Algarve.

Froese, R. y Pauly, D. (Eds). 2015. Fishbase World Wide Web electronic publication. www.fishbase.org.

García-Charton, J.A., Pérez-Ruzafa, A. y Marcos-Diego, C. 2000. Fish visual census methods for detecting gradients of abundance and biomass across boundaries of MPAS. En: Goñi, R., Harmelin-Vivien, M., Badalamenti, F., Le Diréach, L. y Bernard, G. (eds.). Introductory guide to methods for selected ecological studies in marine reserves. GIS Posidonie publication. Marseille, France. pp. 29-34.

Greene, L.E. y Alevizon, W.S. 1989. Comparative accuracies of visual assessment methods for coral reef fishes. Bull. Mar. Scienc. 44: 899-912.

Harmelin-Vivien, M.L., Harmelin, J.G., Chauvet, C., Duval, C., Galzin, R., Lejeune, P., Barnabé, G., Blanc, F., Chevalier, R., Duclerc, J. y Lasserre, G. 1985. Evaluation visuelle des peuplementset populations de poissons: methodes et problemes. Revue D Ecologie - La Terre et la Vie 40: 467-539.

Krajewski, J.P. y Floeter, S.R. 2011. Reef fish community structure of the Fernando de Noronha Archipelago (Equatorial Western Atlantic): the influence of exposure and benthic composition. Environ. Biol. Fish. 92: 25-40. 
Lloris, D., Rucabado, J. y Figeroa, H. 1991. Biogeography of the Macaronesian ichthyofauna. Bol. Mus. Munic. Funchal 43: 191-241.

Longo, G.O. y Floeter, S.R. 2012. Comparison of remote video and diver's direct observations to quantify reef fishes feeding on benthos in coral and rocky reefs. J. Fish Biol. 81: 1773-1780.

Lopez-Jurado, L.F.L. 1998. Inventario preliminar de los recursos naturales litorales de la Republica de Cabo Verde. Las Palmas de Gran Canaria. Gobierno de Canarias. Consejería Política territorial. $181 \mathrm{p}$.

Mapstone, B.D. y Ayling, A.M. 1998. An investigation of optimum methods and unit sizes for the visual estimation of abundances of some coral reef organisms. Great Barrier Reef Marine Park Authority. 70 p.

Margalef, R. 1956. Información y diversidad específica en las comunidades de organismos. Investigación Pesquera. 3: 99-106.

Milazzo, M., Palmeri, A., Falcón, J.M., Badalamenti, F., García-Charton, J.A., Sinopoli, M., Chemello, R. y Brito, A. 2011. Vertical distribution of two sympatric labrid species in the Western Mediterranean and Eastern Atlantic rocky subtidal: local shore topography does matter. Mar. Ecol. 32: 521-531.

Morri, C., Cattaeno-Vietti, R., Sartoni, G. y Banchi, CN. 2000. Shallow epibenthic communities of Ilha do Sal (Cape Verde Archipelago, eastern Atlantic). Arquipélago: Life and Marine Science. Ponta Delgada. Supplement2 (Part A): 157-165.

Sale, P.F. y Sharp, B.J. 1983. Correction for bias in visual transect censuses of coral reef fishes. Coral Reefs. 2: 37-42.

Samoilys, M.A. y Carlos, G. 2000. Determining methods of underwater visual census for estimating the abundance of coral reef fishes. Environ. Biol. Fish. 57: 289-304.

Smith, W.L., Craig, M.T. y Quattro, J.M. 2007. Casting the percomorph net widely: The importance of broad taxonomic sampling in the search for the placement of Serranid and Percid fishes. Copeia. 1: 35-55.

Torres, P.C., Silva, L.C., Serralheiro, A., Mendes, M.H., Macedo, J. y Mota Gomes, A., 2002. Geologia da Ilha do Sal. Serie de Ciencias da Terra. 10: 1-572, Geological Sheets I-II.

VAn der LAnd, J. 1993. Marine biota of the Cape Verde Islands. Courier Forschungsinstitut Senckenberg. 159: 39-44.

Vinnik, L., Silveira, G., Kiselev, S., Farra, V., Weber, M. y Stutzmann, E. 2012. Cape Verde hotspot from the upper crust to the top of the lower mantle. Earth Planet. Scienc. Lett. 259-268.

Watson, R.A., Carlos, G.M. y Samoilys, M.A. 1995. Bias introduced by non-random movements of fish in visual transect surveys. Ecol. Model. 77: 205-214.

Wirtz, P., Brito, A., Falcón, J.M., Freitas, R., Fricke, R., Monteiro, V., Reiner, F. y TarICHE, O. 2013. The coastal fishes of the Cape Verde Islands -new records and an annotated check-list (Pisces). Spixiana. 36: 113-142. 


\section{TABLAS}

\begin{tabular}{|c|c|c|c|}
\hline FAMILIA & ESPECIE & ABUNDANCIA & ABUNDANCIA RELATIVA (\%) \\
\hline Acanthuridae & Acanthurus monroviae & 65 & 1,30 \\
\hline Apogonidae & Apogon imberbis & 92 & 1,83 \\
\hline Aulostomidae & Aulostomus strigosus & 17 & 0,34 \\
\hline Balistidae & Balistes punctatus & 5 & 0,10 \\
\hline \multirow{2}{*}{ Blenniidae } & Ophioblennius atlanticus & 1 & 0,02 \\
\hline & Parablennius salensis * & 26 & 0,52 \\
\hline Carangidae & Caranx crysos & 22 & 0,44 \\
\hline Chaetodontidae & Chaetodon robustus & 4 & 0,08 \\
\hline \multirow{2}{*}{ Diodontidae } & Chilomycterus reticulatus & 1 & 0,02 \\
\hline & Diodon holocanthus & 3 & 0,06 \\
\hline \multirow{2}{*}{ Epinephelidae } & Cephalopholis taeniops & 26 & 0,52 \\
\hline & Epinephelus costae & 1 & 0,02 \\
\hline \multirow{4}{*}{ Gobiidae } & Bathygobius casamancus & 5 & 0,10 \\
\hline & Gnatholepis thompsoni & 57 & 1,14 \\
\hline & Gobius ateriformis * & 3 & 0,06 \\
\hline & Gobius tetrophthalmus * & 624 & 12,44 \\
\hline Haemulidae & Parapristipoma humile * & 680 & 13,55 \\
\hline \multirow{2}{*}{ Holocentridae } & Myripristis jacobus & 13 & 0,26 \\
\hline & Sargocentron hastatum & 38 & 0,76 \\
\hline \multirow{3}{*}{ Labridae } & Bodianus speciosus & 14 & 0,28 \\
\hline & Coris atlantica & 69 & 1,38 \\
\hline & Thalassoma pavo & 262 & 5,22 \\
\hline Labrisomidae & Labrisomus nuchipinnis & 1 & 0,02 \\
\hline Mullidae & Pseudupeneus prayensis & 72 & 1,43 \\
\hline \multirow{3}{*}{ Muraenidae } & Gymnothorax miliaris & 3 & 0,06 \\
\hline & Gymnothorax vicinus & 4 & 0,08 \\
\hline & Muraena melanotis & 2 & 0,04 \\
\hline Pomacanthidae & Holacanthus africanus & 9 & 0,18 \\
\hline \multirow{7}{*}{ Pomacentridae } & Abudefduf hoefleri & 6 & 0,12 \\
\hline & Similiparma lurida & 1 & 0,02 \\
\hline & Abudefdufsaxatilis & 9 & 0,18 \\
\hline & Chromis lubbocki* & 1263 & 25,17 \\
\hline & Chromis multilineata & 922 & 18,37 \\
\hline & Similiparma hermani ${ }^{*}$ & 4 & 0,08 \\
\hline & Stegastes imbricatus & 358 & 7,13 \\
\hline
\end{tabular}




\begin{tabular}{|c|c|c|c|}
\hline \multirow{2}{*}{ Priacanthidae } & Heteropriacanthus cruentatus & 11 & 0,22 \\
\hline & Priacanthus arenatus & 13 & 0,26 \\
\hline \multirow{3}{*}{ Scaridae } & Scarus hoefleri & 26 & 0,52 \\
\hline & Sparisoma choati & 4 & 0,08 \\
\hline & Sparisoma cretense & 174 & 3,47 \\
\hline \multirow{2}{*}{ Scorpaenidae } & Scorpaena laevis & 3 & 0,06 \\
\hline & Scorpaena maderensis & 1 & 0,02 \\
\hline Serranidae & Rypticus aff.saponaceus & 4 & 0,08 \\
\hline \multirow{5}{*}{ Sparidae } & Diplodus fasciatus * & 23 & 0,46 \\
\hline & Diplodus sargus lineatus * & 24 & 0,48 \\
\hline & Diplodus prayensis * & 15 & 0,30 \\
\hline & Diplodus puntazzo & 3 & 0,06 \\
\hline & Virididentex acromegalus * & 1 & 0,02 \\
\hline Synodontidae & Synodus synodus & 2 & 0,04 \\
\hline \multirow{3}{*}{ Tetraodontidae } & Canthigaster capistrata & 30 & 0,60 \\
\hline & Sphoeroides marmoratus & & \\
\hline & 2 & 0,04 & \\
\hline Total familias: 24 & Total sp: 50 & Total ind: 5018 & 100 \\
\hline
\end{tabular}

TABLA 2. A) RESULTADOS DEL ANÁLISIS PERMANOVA EN EL QUE SE CONTRASTAN LAS VARIACIONES EN LA RIQUEZA DE ESPECIES EN FUNCIÓN DEL MÉTODO UTILIZADO (TRANSECTO, CIRCULAR), LA LOCALIDAD MUESTREADA (1-8) Y LA INTERACCIÓN ENTRE AMBOS FACTORES. LOS VALORES SIGNIFICATIVOS SE MUESTRAN EN NEGRITA. B) ANÁLISIS A POSTERIORI DE LAS COMPARACIONES POR PARES DEL FACTOR «MÉTODO» CON CADA UNA DE LAS LOCALIDADES. LOS VALORES SIGNIFICATIVOS $(\mathrm{P}<0,05)$ SE MUESTRAN EN NEGRITA

Presencia/ausencia de especies

\begin{tabular}{llllll}
\hline a) Fuente de variación & df & SS & MS & Pseudo- F & P (perm) \\
\hline Método & 1 & 4446,9 & 4446,9 & 2,064 & 0,079 \\
\hline Localidad & 7 & 79284 & 11326 & 7,906 & $\mathbf{0 , 0 0 0}$ \\
\hline MétodoxLocalidad & 7 & 15104 & 2157,7 & 1,506 & $\mathbf{0 , 0 2 5}$ \\
\hline Residual & 58 & 83091 & 1432,6 & & \\
\hline Total & 73 & $1,9185 \mathrm{E} 5$ & & & \\
\hline
\end{tabular}

b) Análisis a posteriori

\begin{tabular}{lcccc}
\hline & $\mathrm{t}$ & $\mathrm{P}($ perm) & Unique perms \\
\hline Método x Localidad 1 & 1,345 & 0,159 & 63 \\
\hline Método x Localidad 2 & 1,932 & $\mathbf{0 , 0 1 1}$ & 84 \\
\hline Método x Localidad 3 & 1,235 & 0,181 & 84 \\
\hline Método x Localidad 4 & 1,458 & 0,053 & 210 \\
\hline
\end{tabular}




\begin{tabular}{lllll}
\hline Método x Localidad 5 & 1,154 & 0,356 & 84 \\
\hline Método x Localidad 6 & 0,604 & 0,906 & 84 \\
\hline Método x Localidad 7 & 1,576 & 0,065 & 63 \\
\hline Método x Localidad 8 & 0,985 & 0,473 & 53 \\
\hline
\end{tabular}

\begin{tabular}{|c|c|c|c|c|c|c|c|c|c|c|c|c|c|c|}
\hline $\begin{array}{r}\text { TABLA 3. RESUME } \\
\text { EN EL QUE } \\
\text { LA DISI } \\
\text { EN NEGRITA I } \\
\text { Y P }\end{array}$ & $\begin{array}{l}\text { E MU } \\
\text { MILIT } \\
\text { AS ES } \\
\text { RESEN }\end{array}$ & $\begin{array}{l}\text { LANÁ } \\
\text { JESTH } \\
\text { TUD E } \\
\text { SPECI } \\
\text { NTAN }\end{array}$ & $\begin{array}{l}\text { RAN I } \\
\text { ENTR } \\
\text { ES QL } \\
\text { J POR }\end{array}$ & $\begin{array}{l}\text { LAS C } \\
\text { E LO } \\
\text { JE SE } \\
\text { CEN }\end{array}$ & $\begin{array}{l}\text { CONT } \\
\text { CALI } \\
\text { REPI } \\
\text { TAJES }\end{array}$ & $\begin{array}{l}\text { TRIBU } \\
\text { IDADE } \\
\text { ITEN } \\
\text { S DE C }\end{array}$ & $\begin{array}{l}\text { JCION } \\
\text { ES. AP } \\
\text { A LO } \\
\text { CONT }\end{array}$ & $\begin{array}{l}\text { JES (\% } \\
\text { AREC } \\
\text { LARG } \\
\text { RIBU }\end{array}$ & $\begin{array}{l}\text { 6) DE } \\
\text { CEN D } \\
\text { OO DF } \\
\text { CIÓN }\end{array}$ & $\begin{array}{l}\text { CAD } \\
\text { DESTA } \\
\text { E LOS } \\
\text { N ALT }\end{array}$ & $\begin{array}{l}\text { A ESP } \\
\text { ACAD } \\
\text { RESL } \\
\text { OS }\end{array}$ & $\begin{array}{l}\text { ECIE } \\
\text { AS } \\
\text { ULTAI }\end{array}$ & DOS & \\
\hline Especies & & & & & ONTRI & IBUCIÓ & N & ENTRE & LOCA & LIDAD & & & & \\
\hline & $1-2$ & $1-3$ & $1-4$ & $1-5$ & $1-6$ & $1-7$ & $1-8$ & $2-3$ & $2-4$ & $2-5$ & $2-6$ & $2-7$ & $2-8$ & $3-4$ \\
\hline Chromis lubbocki & 8,73 & 5,15 & 7,71 & 9,82 & & & 18,78 & 5,57 & & & 8,01 & 9,29 & 11,75 & 5,28 \\
\hline Stegastes imbricatus & 8,63 & 4,51 & 5,59 & 7,58 & & 13,44 & & 5,78 & 3,85 & 4,23 & 7,88 & & & 4,75 \\
\hline Gobius tetrophthalmus & 7,58 & & 4,45 & & & 10,89 & 15,49 & 7,22 & 4,92 & 7,53 & 7,78 & & & 3,91 \\
\hline Thalassoma pavo & 6,54 & 7,88 & 3,63 & 8,38 & 7,36 & 13,44 & & 4,26 & 4,73 & 4,69 & 5,14 & 3,85 & 6,01 & 4,62 \\
\hline Gnatholepis thompsoni & & 8,45 & & & 7,55 & & & 4,88 & & 4,06 & 4,11 & 3,91 & & 5,13 \\
\hline Parablennius salensis & & 7,71 & 5,37 & 6,74 & 10,81 & & 9,74 & & & & & & & \\
\hline Pseudupeneus prayensis & 3,27 & 7,20 & 4,30 & 6,70 & 14,30 & & & 4,57 & 4,06 & 5,46 & 5,70 & 5,56 & 3,90 & 4,55 \\
\hline Sargocentron hastatum & 5,95 & 4,51 & 4,19 & 5,06 & & & & 4,87 & 4,46 & 5,51 & 5,96 & 7,39 & 6,78 & 4,29 \\
\hline Cephalopholis taeniops & & 4,89 & 5,28 & 4,88 & 4,62 & & & 3,58 & 4,75 & 4,52 & & & & 4,55 \\
\hline Acanthurus monroviae & & & 4,74 & & & & & & 4,28 & & & & & 4,37 \\
\hline Chromis multilineata & 3,12 & & 5,41 & & & 10,89 & 18,78 & 3,44 & 4,56 & 4,69 & 3,11 & 7,10 & 7,94 & 4,81 \\
\hline Canthigaster capistrata & 3,95 & & & 5,17 & 7,98 & & & 3,91 & 3,82 & 5,15 & 4,62 & 5,10 & 4,83 & \\
\hline Aulostomus strigosus & 3,28 & & & & & & & 2,97 & 3,40 & & 3,22 & 3,96 & 4,27 & \\
\hline Sparisoma cretense & 6,22 & 5,97 & 5,78 & 7,53 & 9,86 & 11,16 & & 4,57 & 4,07 & 4,78 & 4,74 & 4,26 & 7,94 & 4,58 \\
\hline Scarus hoefleri & & & & & & & & & 3,96 & & & 4,29 & 3,81 & \\
\hline Coris atlantica & & & & & & & & 3,11 & 2,97 & 4,17 & & & & \\
\hline Bodianus speciosus & & 4,33 & & & & & & 3,67 & & & & & & 3,54 \\
\hline Diplodus fasciatus & & & 4,87 & & & & & & 4,27 & & & & & 4,44 \\
\hline Holacanthus africanus & & & & & & & & & 3,02 & & & & & \\
\hline Abudefduf hoefleri & & & & & & & & & & & & & 3,72 & \\
\hline
\end{tabular}

Continuación tabla 3

\begin{tabular}{|c|c|c|c|c|c|c|c|c|c|c|c|c|c|c|}
\hline \multirow[t]{2}{*}{ EsPeCIES } & \multicolumn{14}{|c|}{ CONTRIBUCIÓN (\%) ENTRE LOCALIDADES } \\
\hline & $3-5$ & $3-6$ & $3-7$ & $3-8$ & $4-5$ & $4-6$ & $4-7$ & $4-8$ & $5-6$ & $5-7$ & $5-8$ & $6-7$ & $6-8$ & $7-8$ \\
\hline Chromis lubbocki & 6,74 & 5,18 & 4,70 & & 4,78 & 6,95 & 7,09 & 8,98 & 9,84 & 9,20 & 10,92 & 12,33 & & 4,74 \\
\hline Stegastes imbricatus & 6,20 & 4,56 & 7,95 & 8,63 & 4,53 & 5,25 & 4,30 & 4,60 & 7,86 & & & & 13,25 & \\
\hline Gobius tetrophthalmus & & & 9,61 & 10,72 & 5,39 & 4,14 & 5,63 & 5,90 & & 9,43 & 9,43 & 11,34 & 12,40 & \\
\hline Thalassoma pavo & 5,47 & 6,90 & 5,11 & 6,20 & & 4,45 & 6,28 & 5,18 & 7,11 & & 6,53 & 8,27 & 6,87 & 12,55 \\
\hline
\end{tabular}




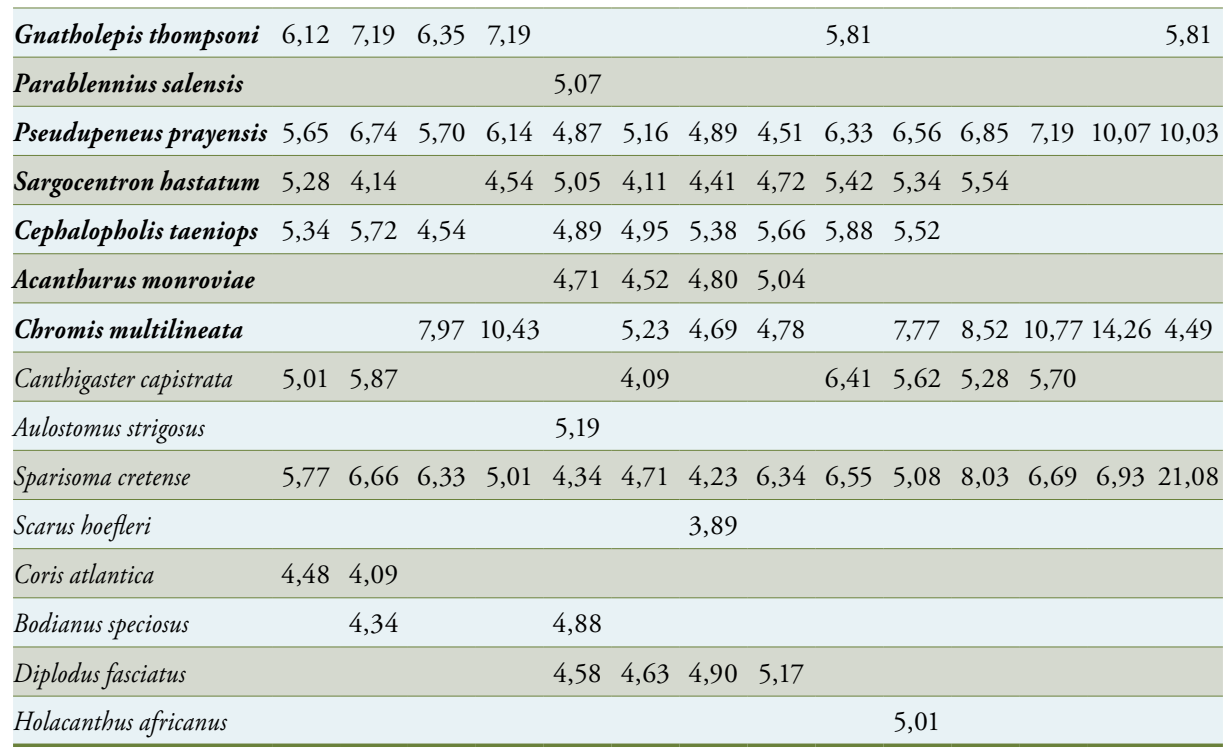

TABLA 4. ANÁLISIS SIMPER PARA LA LOCALIDAD 2, EN LA QUE SE MUESTRAN LAS NUEVE ESPECIES QUE MÁS CONTRIBUYEN A DIFERENCIAR LOS MÉTODOS ENTRE SÍ

\begin{tabular}{lcc}
\multicolumn{1}{c}{ Especies } & Contribución\% & Acumulado\% \\
\hline Chromis multilineata & 8,89 & 8,89 \\
\hline Thalassoma pavo & 8,89 & 17,78 \\
\hline Balistes punctatus & 5,93 & 23,71 \\
\hline Coris atlantica & 5,93 & 29,64 \\
\hline Abudefdufhoefleri & 5,47 & 35,10 \\
\hline Scarus hoefleri & 5,44 & 40,55 \\
\hline Sparisoma cretense & 5,44 & 45,99 \\
\hline Canthigaster capistrata & 5,03 & 51,02 \\
\hline Sargocentron hastatum & 4,82 & 55,84 \\
\hline
\end{tabular}

TABLA 5. (A) RESULTADOS OBTENIDOS PARA EL ANÁLISIS ESPECÍFICO DONDE SE PONE DE MANIFIESTO LA DIFERENCIA ENTRE EL MÉTODO DE TRANSECTO Y EL CIRCULAR, EN CUANTO A LA RIQUEZA DE ESPECIES (S), EL NÚMERO (N) Y ÍNDICES DE DIVERSIDAD DE MARGALEF [D= (S-1)/LNN]. (B) LISTADO DE ESPECIES EXCLUSIVAMENTE REGISTRADAS POR UN MÉTODO Y POR EL OTRO

a) ANÁLisis ESPeCífico - RIQUEZA

\begin{tabular}{lccc}
\hline Método & S & N & d \\
\hline Transecto & $\mathbf{4 0}$ & 59 & 9,56 \\
\hline Circular & $\mathbf{4 5}$ & 71 & 10,31 \\
\hline
\end{tabular}




\begin{tabular}{|c|c|}
\hline \multicolumn{2}{|l|}{ в) Especies } \\
\hline Transecto & Circular \\
\hline \multicolumn{2}{|r|}{ Caranx crysos } \\
\hline & Chilomycterus reticulatus \\
\hline Diplodus puntazzo & Diodon holocanthus \\
\hline Gobius ateriformis & Epinephelus costae \\
\hline Labrisomus nuchipinnis & Ophioblennius atlanticus \\
\hline Scorpaena maderensis & Priacanthus arenatus \\
\hline Sphoeroides marmoratus & Scorpaena laevis \\
\hline & Similiparma lurida \\
\hline & Synodus synodus \\
\hline & Virididentex acromegalus \\
\hline
\end{tabular}

TABLA 6. A) RESULTADOS DEL ANÁLISIS PERMANOVA EN EL QUE SE CONTRASTAN LAS VARIACIONES EN LA ABUNDANCIA DE ESPECIES SEGÚN EL MÉTODO DE MUESTREO (TRANSECTO, CIRCULAR), LA LOCALIDAD (1-8) Y LA INTERACCIÓN ENTRE ESTOS DOS FACTORES. B) ANÁLISIS A POSTERIORI DE LAS COMPARACIONES POR PARES DEL FACTOR «MÉTODO» CON CADA UNA DE LAS LOCALIDADES. LOS VALORES SIGNIFICATIVOS SE MUESTRAN EN NEGRITA. LOS VALORES SIGNIFICATIVOS $(P<0,05)$ SE MUESTRAN EN NEGRITA

Abundancia de especies

\begin{tabular}{lccccc}
\hline a) Fuente de variación & df & SS & MS & PsEUDO- F & P (PERM) \\
\hline Método & 1 & 4067,6 & 4067,6 & 1,6208 & 0,139 \\
\hline Localidad & 7 & 83375 & 11911 & 8,0394 & $\mathbf{0 , 0 0 0}$ \\
\hline Método x Localidad & 7 & 17605 & 2515,1 & 1,6976 & $\mathbf{0 , 0 0 1}$ \\
\hline Residual & 58 & 85929 & 1481,5 & & \\
\hline Total & 73 & $2,0434 \mathrm{E} 5$ & & & \\
\hline
\end{tabular}

b) ANÁLISIS A POSTERIORI

\begin{tabular}{lccc} 
& $\mathrm{t}$ & $P(P E R M)$ & UNIQUE PERMS \\
\hline Método x Localidad 1 & 1,494 & 0,061 & 84 \\
\hline Método x Localidad 2 & 1,989 & $\mathbf{0 , 0 1 1}$ & 84 \\
\hline Método x Localidad 3 & 1,412 & 0,091 & 84 \\
\hline Método x Localidad 4 & 1,294 & 0,113 & 210 \\
\hline Método x Localidad 5 & 1,001 & 0,432 & 84 \\
\hline Método x Localidad 6 & 0,966 & 0,556 & 84 \\
\hline Método x Localidad 7 & 1,477 & 0,048 & 84 \\
\hline Método x Localidad 8 & 1,001 & 0,469 & 210 \\
\hline
\end{tabular}




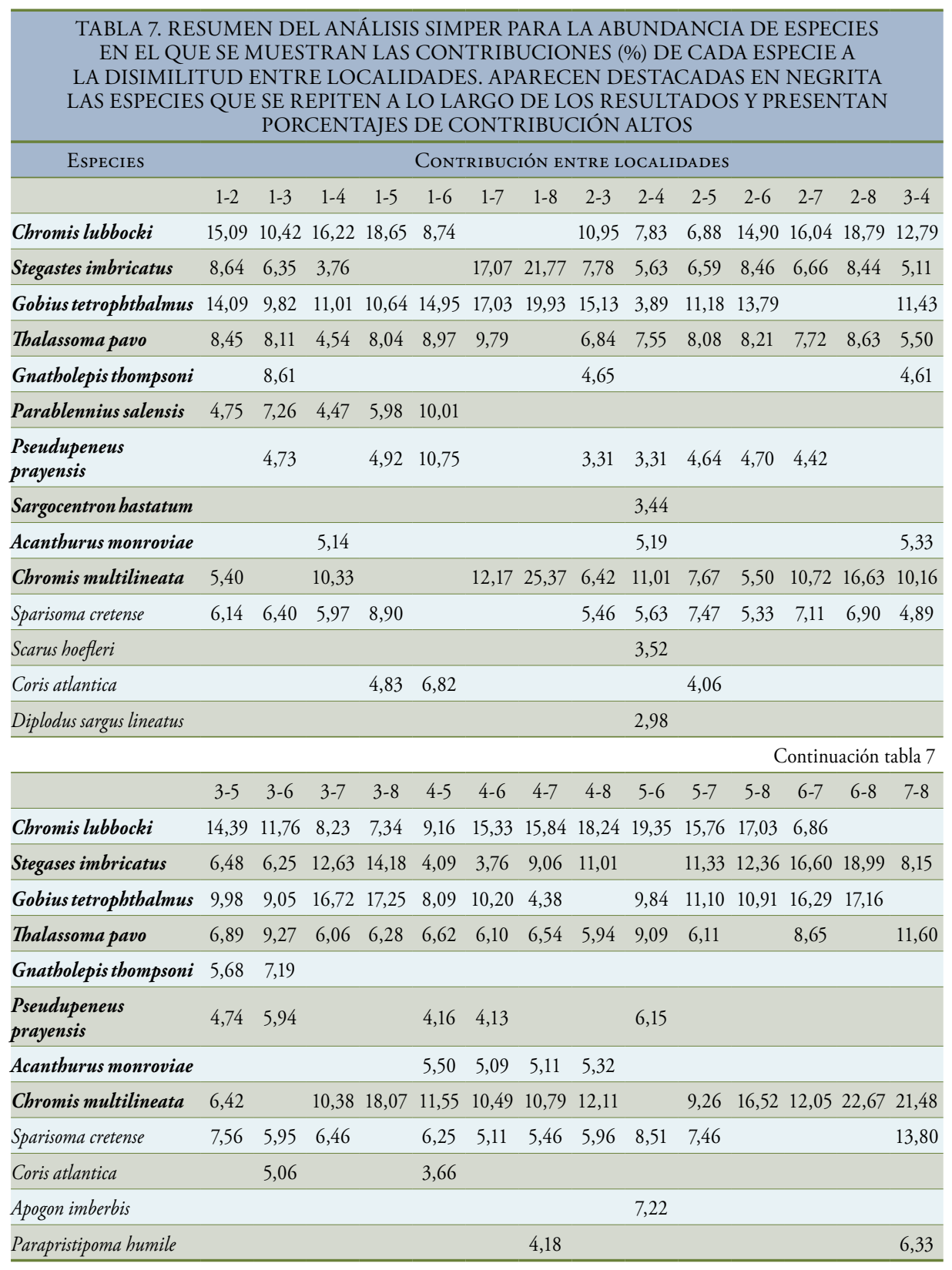




\begin{tabular}{lcc}
$\begin{array}{l}\text { TABLA 8. SIMPER DE LA LOCALIDAD } \\
\text { QUE MÁS CONTRIBUYEN A DIFERENCIAR LOS MÉTODOS ENTRE SÍ }\end{array}$ & ACUMULADO\% \\
\hline \multicolumn{1}{c}{ EsPECIES } & CONTRIBUCIÓN\% & 17,58 \\
\hline Chromis multilineata & 17,58 & 30,34 \\
\hline Thalassoma pavo & 12,76 & 37,26 \\
\hline Sparisoma cretense & 6,92 & 42,24 \\
\hline Coris atlantica & 4,98 & 46,51 \\
\hline Stegastes imbricatus & 4,27 & 50,70 \\
\hline Aulostomus strigosus & 4,20 & 54,77 \\
\hline Scarus hoefleri & 4,07 & 58,76 \\
\hline Sargocentron hastatum & 3,99 & \\
\hline
\end{tabular}

TABLA 9. NIVELES DE SIGNIFICACIÓN OBTENIDOS PARA CADA UNA DE LAS 8 LOCALIDADES EN RELACIÓN A LAS DIFERENCIAS EN LAS ESTIMAS ENTRE MÉTODOS DE MUESTREO Y LA DIVERSIDAD, SEGÚN EL ÍNDICE DE MARGALEF [D= (S-1)/LNN], DE CADA LOCALIDAD

\begin{tabular}{ccc}
\hline LOCALIDAD & Nivel SIGNIFICACIÓN & $\mathrm{d}$ \\
\hline 1 & 0,061 & 2,934 \\
\hline $\mathbf{2}$ & $\mathbf{0 , 0 1 1}$ & $\mathbf{7 , 1 4 5}$ \\
\hline 3 & 0,091 & 6,434 \\
\hline 4 & 0,113 & 6,188 \\
\hline 5 & 0,432 & 4,69 \\
\hline 6 & 0,556 & 4,263 \\
\hline 7 & 0,048 & 3,656 \\
\hline 8 & 0,469 & 2,682 \\
\hline
\end{tabular}




\section{FIGURAS}

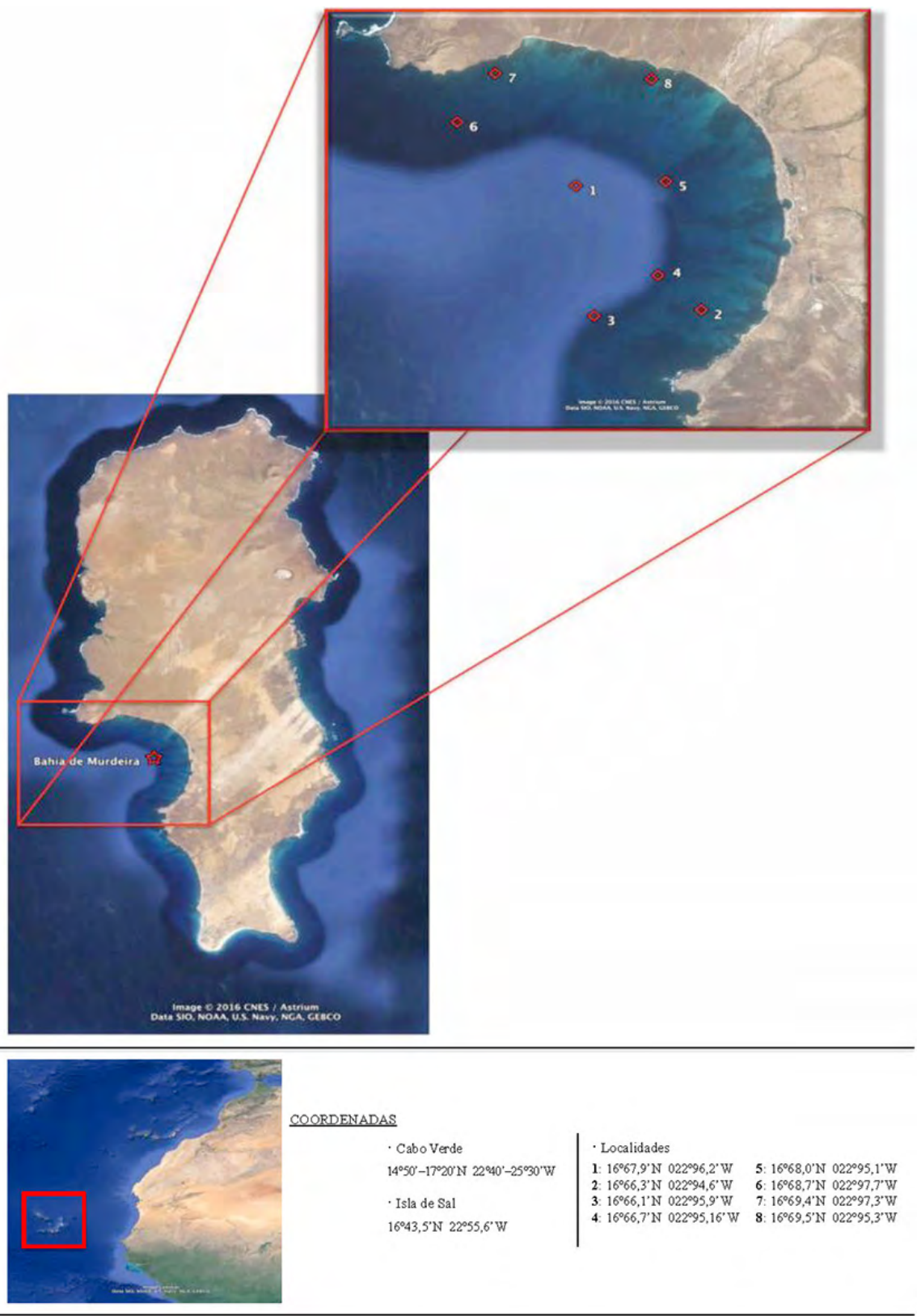

Figura 1. Localización de la Bahía de Murdeira, al suroeste de la isla de Sal, Cabo Verde y detalle de la posición de las ocho localidades donde se llevó a cabo el estudio. 
a)

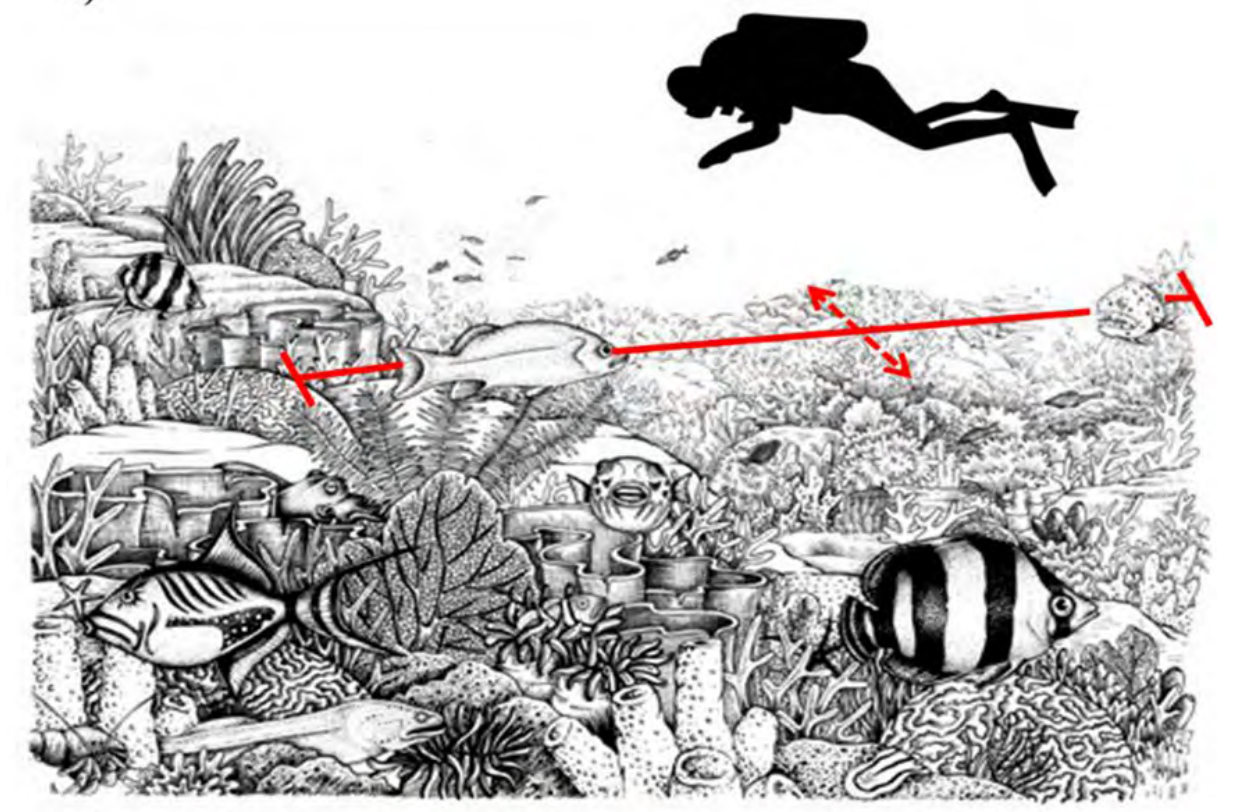

b)

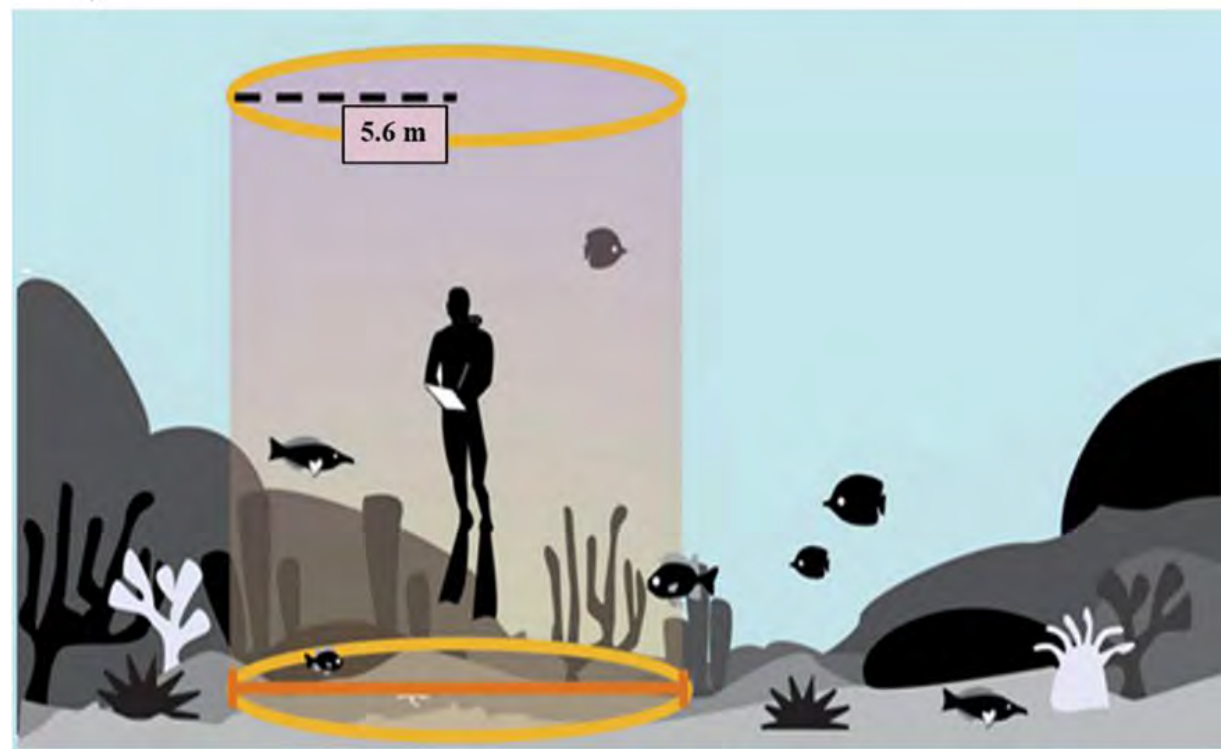

Figura 2. (a). Técnica de muestreo visual por «Transecto», las flechas discontinuas indican la anchura del área de muestreo ( 2 metros en total) y la delimitación de longitud marca los $20 \mathrm{~m}$ de distancia a muestrear. (b) Técnica de muestreo visual de tipo de «Punto fijo» o «Circular», la línea discontínua marca el radio de la circunferencia imaginaria a muestrear. 

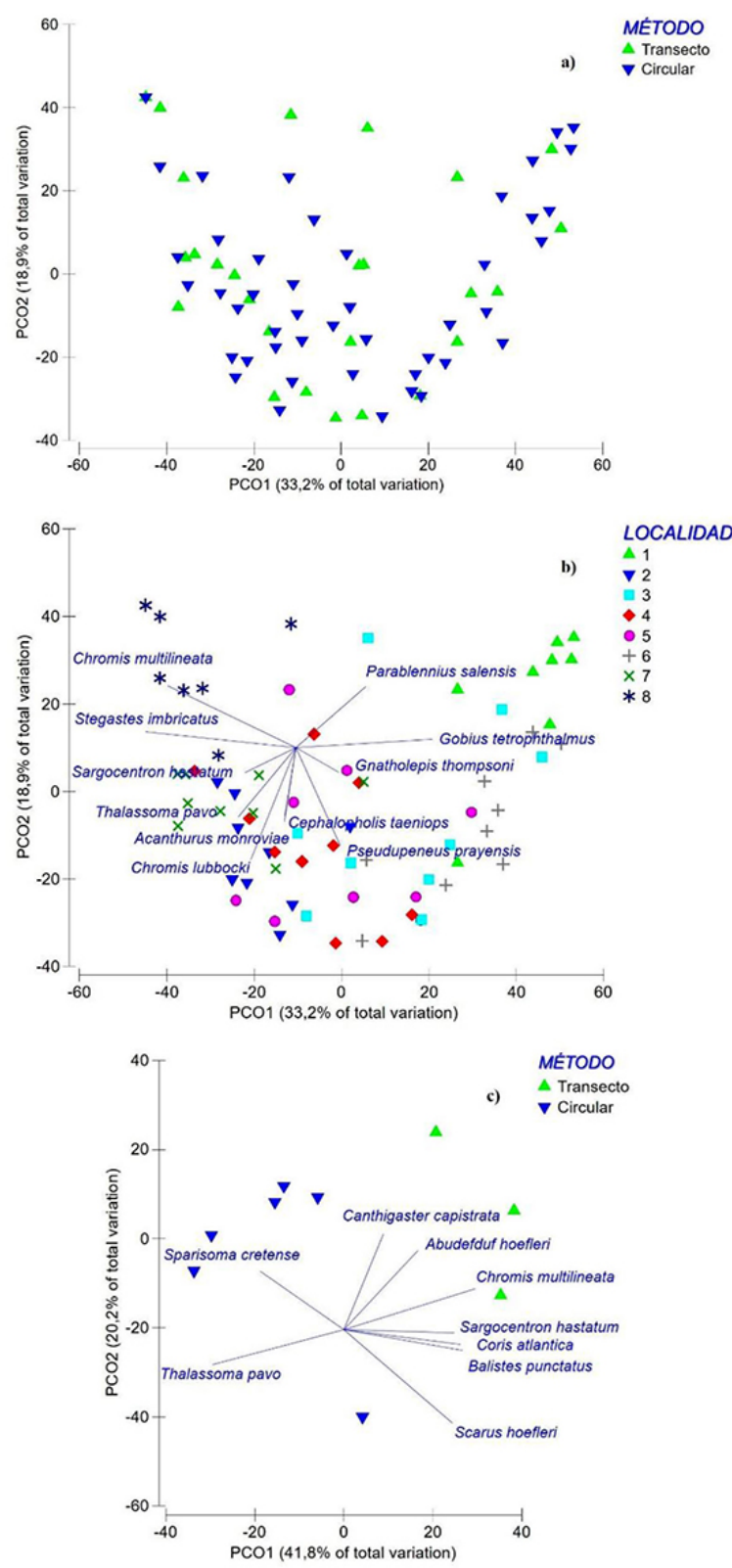

Figura 3. Análisis de ordenación PCO para la variable riqueza en el que se representa el factor Método (a); el factor Localidad, incluyendo vectores superpuestos que representan a las once especies que según el análisis SIMPER contribuyen en mayor medida a las diferencias entre localidades (b); y una ordenación parcial de los muestreos de la localidad 2 para representar las diferencias encontradas entre ambos métodos en base a las especies muestreadas.

De la misma forma, los vectores representan las nueve especies que marcaron las diferencias entre los métodos (c). 

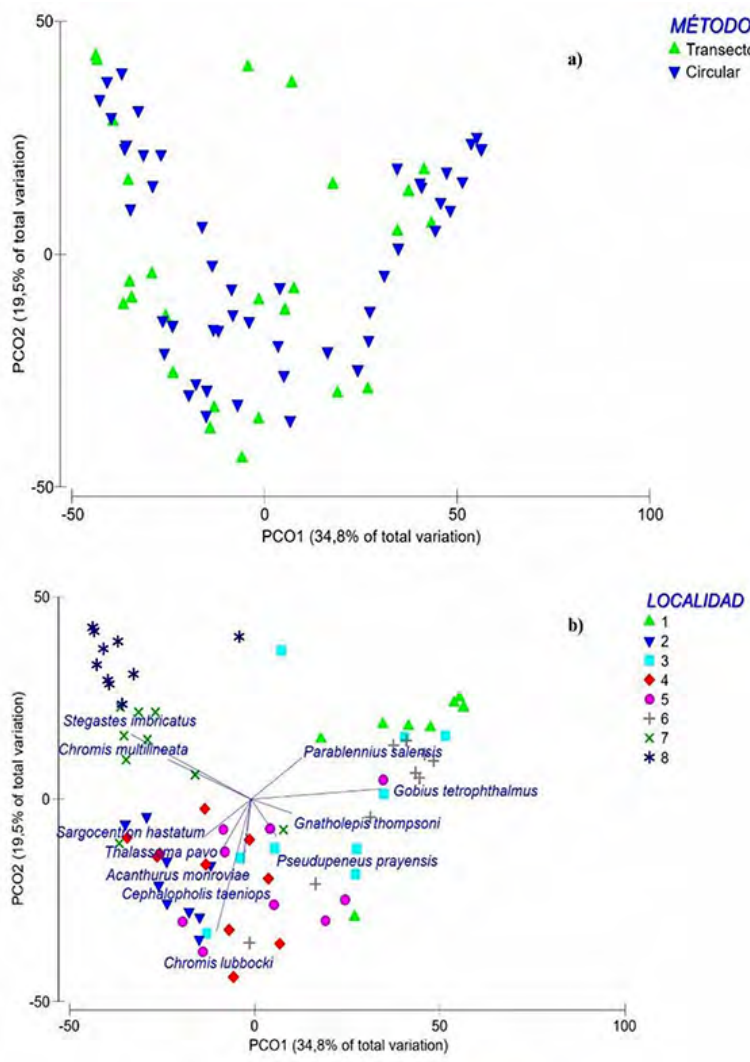

MÉTODO

$\checkmark$ Circular

O1 ( $34,8 \%$ of total variation)

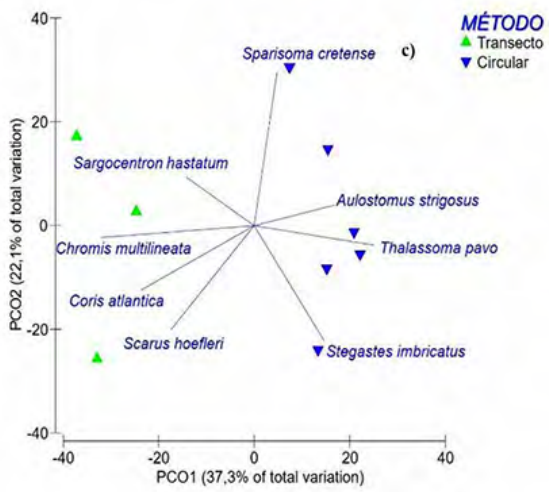

Figura 4. Análisis de ordenación PCO para la variable abundancia en el que se representa el factor Método (a); el factor Localidad, incluyendo vectores superpuestos que representan las once especies que según el análisis SIMPER contribuyen en mayor medida a las diferencias entre localidades

(b); y una ordenación parcial de los muestreos de la localidad 2 para representar las diferencias encontradas entre ambos métodos en base a las especies muestreadas. De la misma forma, los vectores representan las ocho especies que marcaron las diferencias entre los métodos (c). 


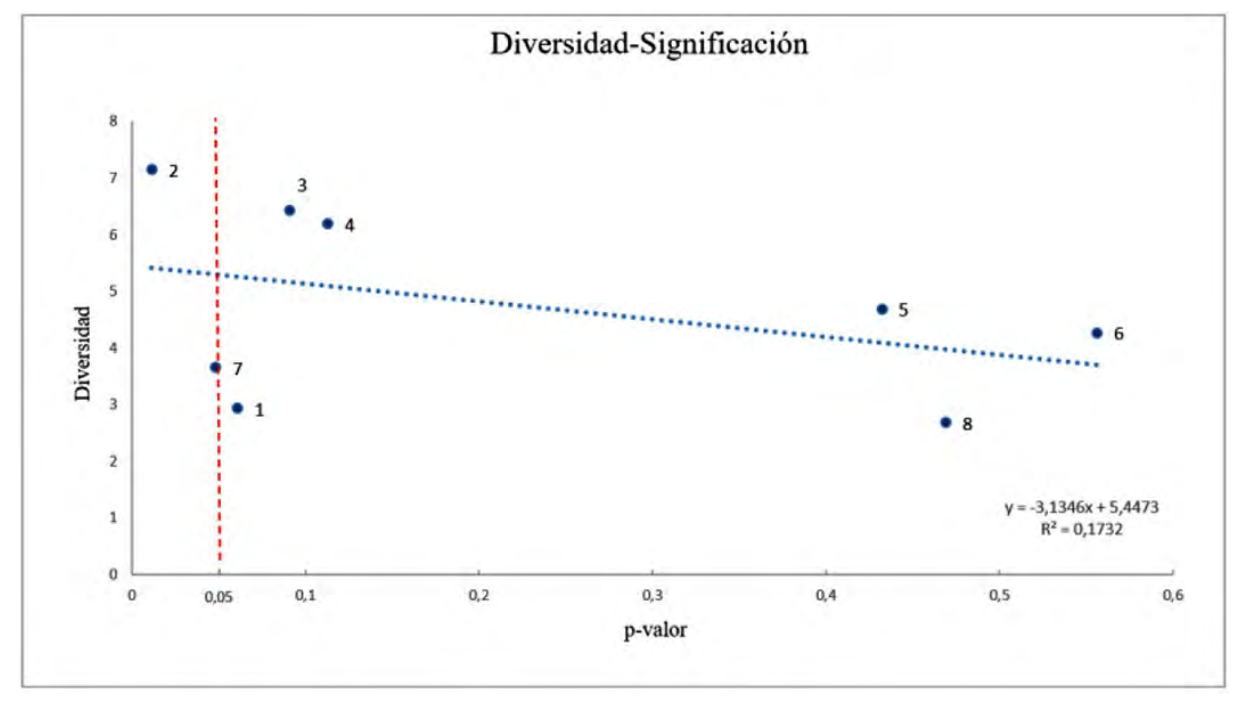

Figura 5. Relación entre la diversidad de cada localidad (y) y el p-valor entre métodos de muestreo (x). Cuanto mayor es la diversidad de la zona menor es el p-valor, es decir cuanto mayor es la diversidad íctica mayores son las diferencias entre métodos.

La línea roja marca el nivel de significación $\mathrm{p}=0,05$. 


\section{ANEXO 1}

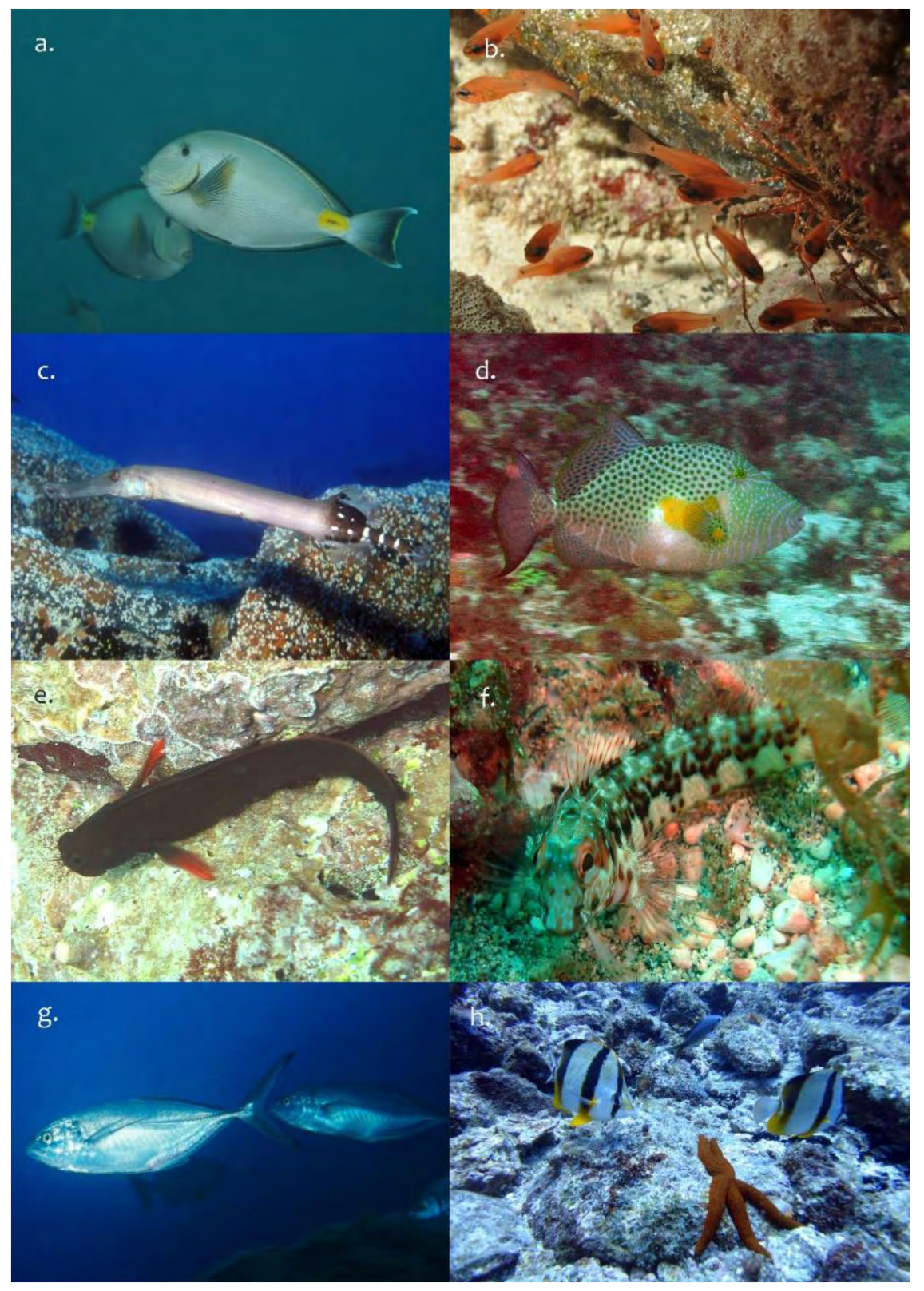

Lámina 1. a. Acanthurus monroviae. b. Apogon imberbis. c. Aulostomus strigosus (Foto tomada en Canarias). d. Balistes punctatus. e. Ophioblennius atlanticus. f. Parablennius salensis (endémico). g. Caranx crysos (Foto J.M. Falcón, tomada en Canarias). h. Chaetodon robustus. 


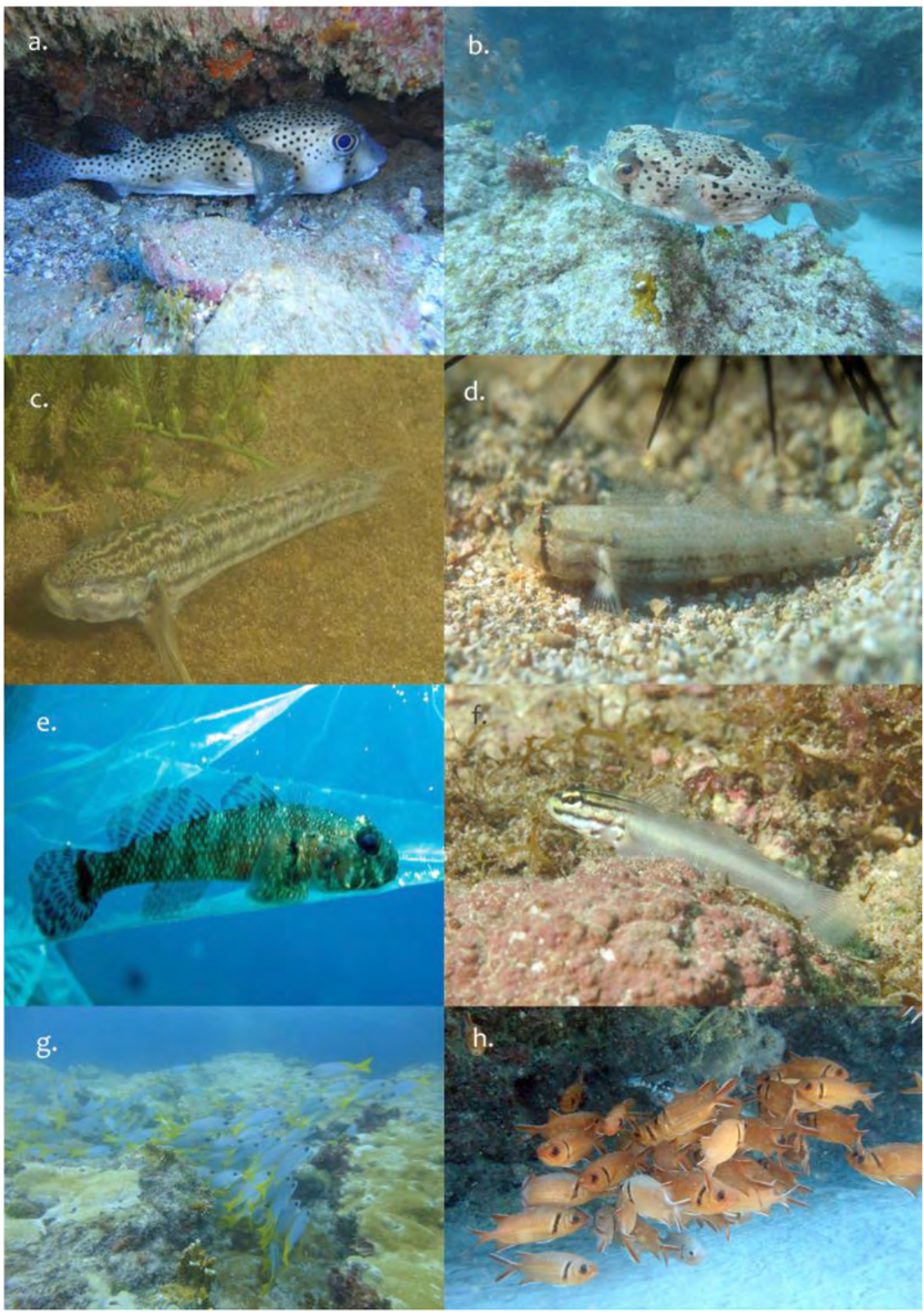

Lámina 2. a. Chilomycterus reticulatus. b. Diodon holocanthus. c. Bathygobius casamancus. d. Gnatholepis thompsoni (Foto tomada en Canarias). e. Gobius ateriformis (endémico, foto P. Wirtz). f. Gobius tetrophthalmus (endémico). g. Parapristipoma humile (endémico). h. Myripristis jacobus. 

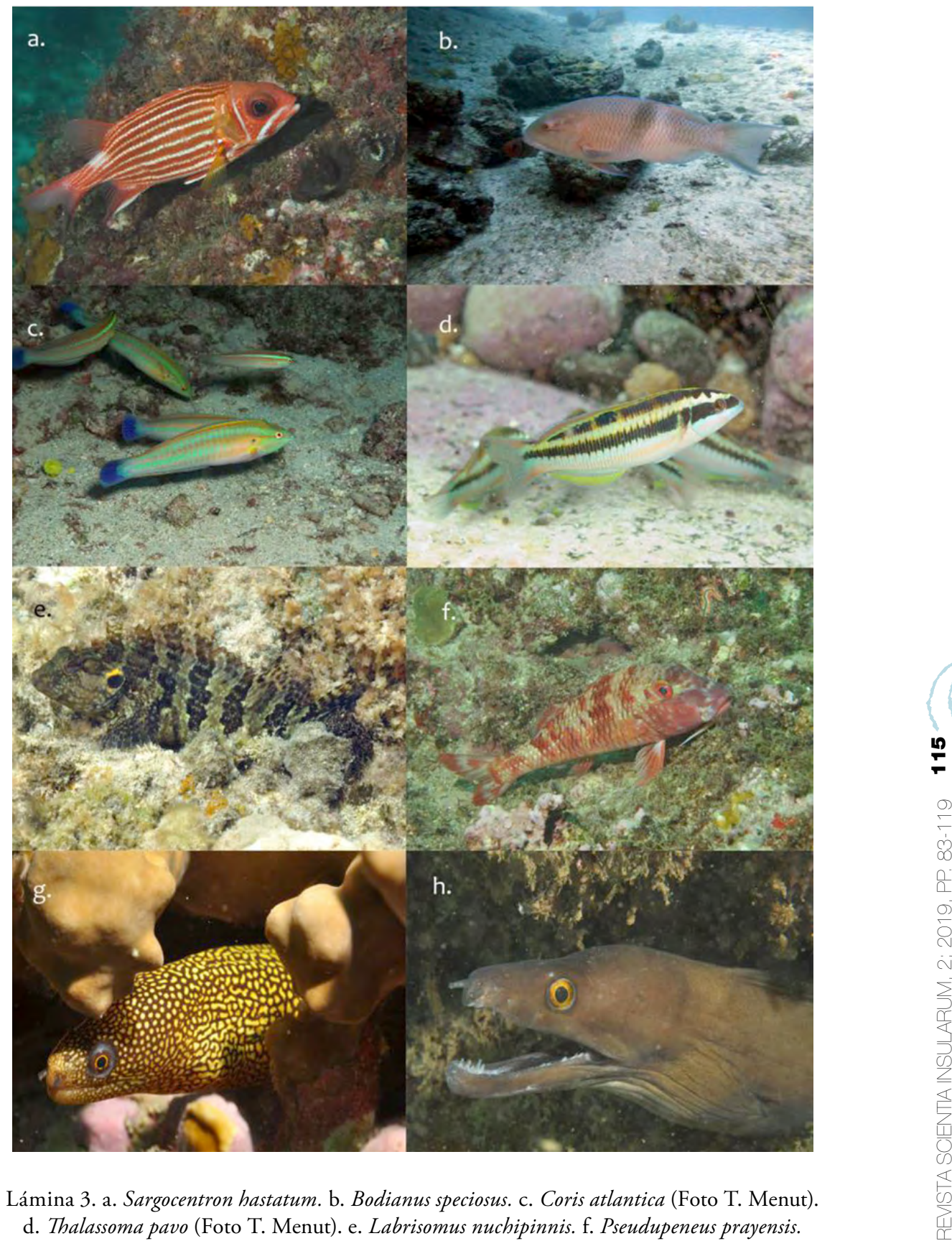

Lámina 3. a. Sargocentron hastatum. b. Bodianus speciosus. c. Coris atlantica (Foto T. Menut). d. Thalassoma pavo (Foto T. Menut). e. Labrisomus nuchipinnis. f. Pseudupeneus prayensis. g. Gymnothorax miliaris. h. Gymnothorax vicinus. 


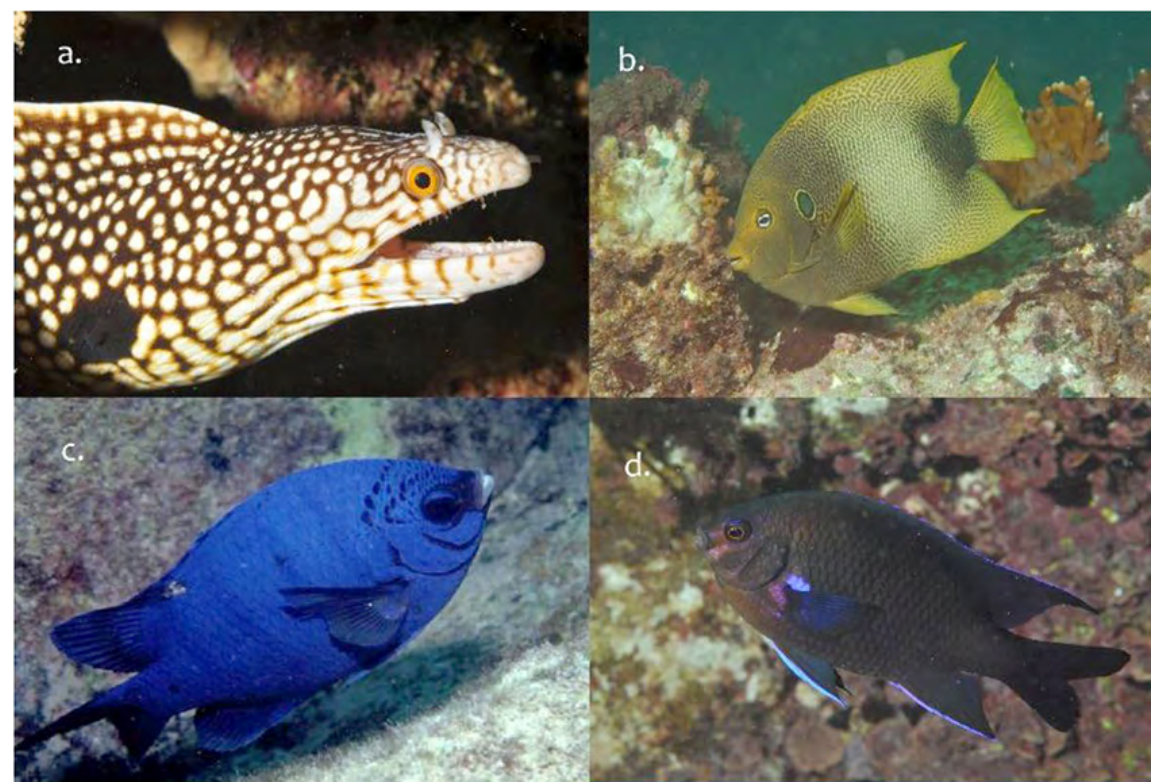

e.
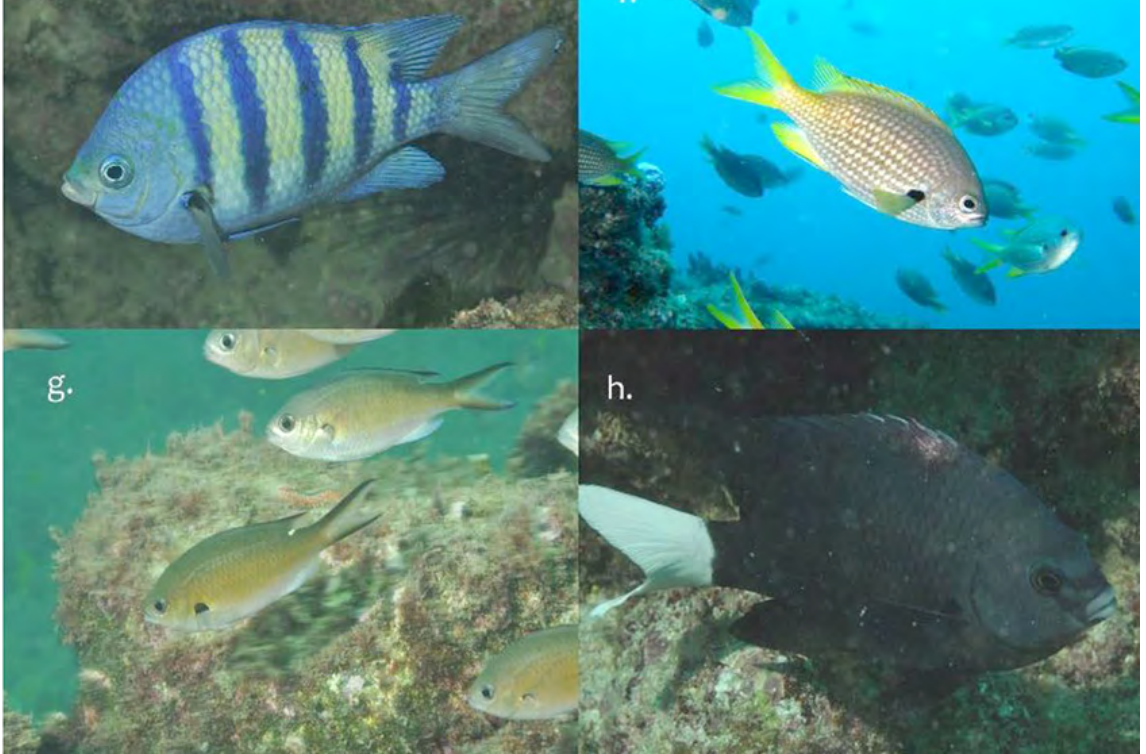

Lámina 4. a. Muraena melanotis. b. Holacanthus africanus. c. Abudefdufhoefleri. d. Similiparma lurida (Foto tomada en Canarias). e. Abudefduf saxatilis. f. Chromis lubbocki (endémico). g. Chromis multilineata. h. Similiparma hermani (endémico). 


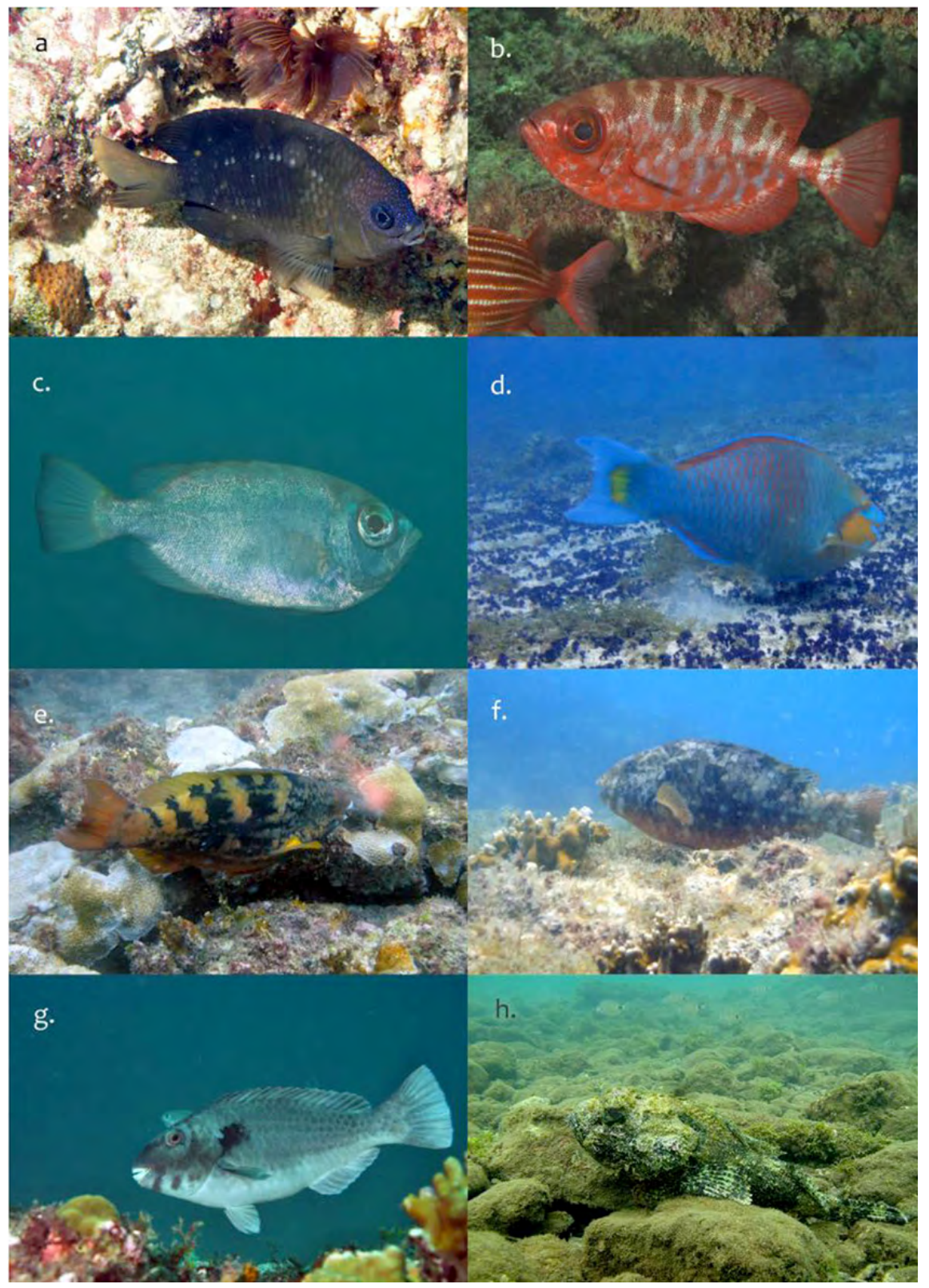

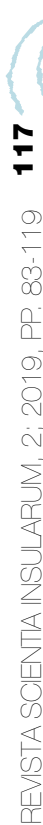

Lámina 5. a. Stegastes imbricatus. b. Heteropriacanthus cruentatus. c. Priacanthus arenatus. d. Scarus hoefleri macho. e. Scarus hoefleri hembra. f. Sparisoma choati. g. Sparisoma cretense. h. Scorpaena laevis. 


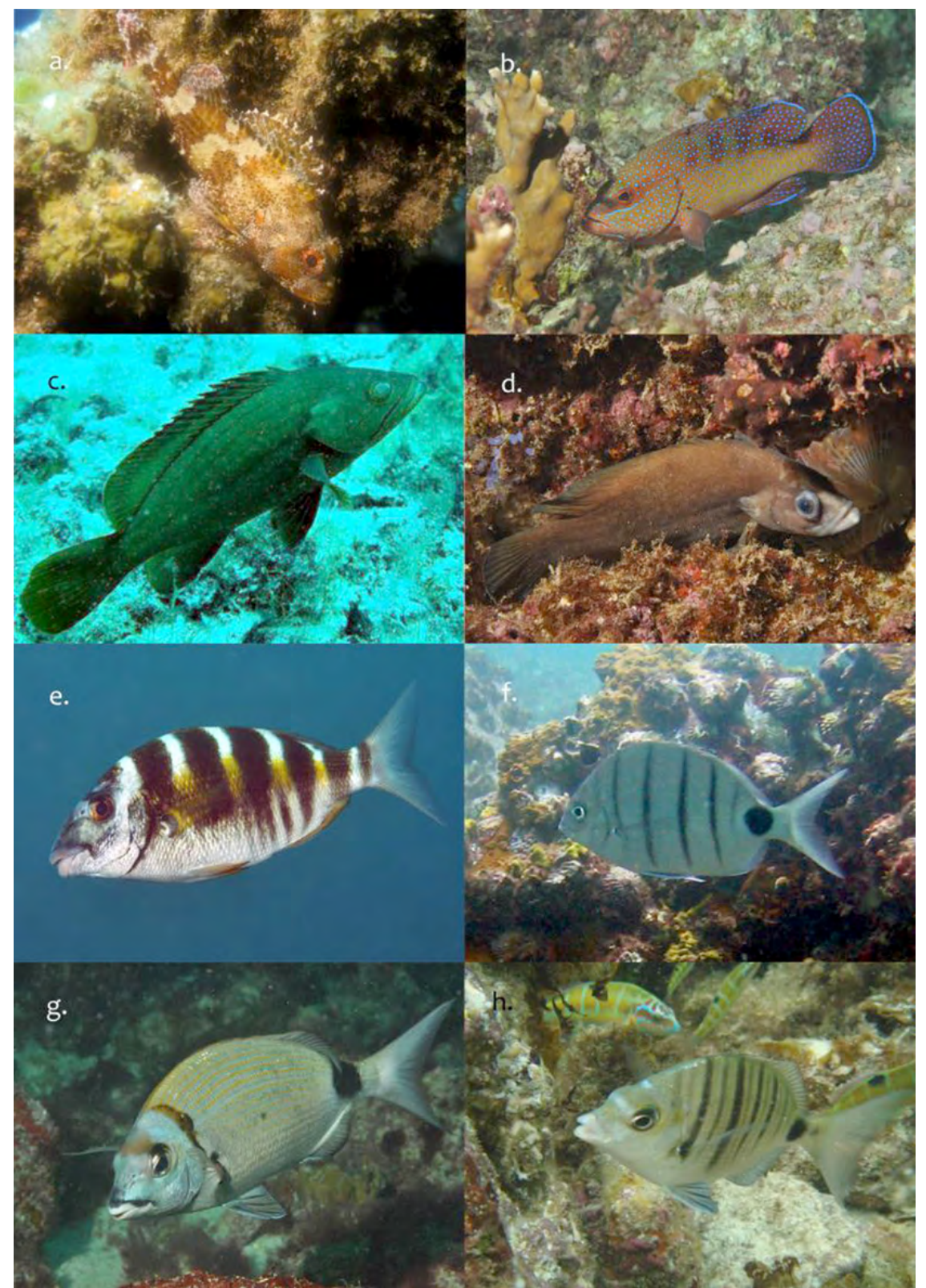

Lámina 6. a. Scorpaena maderensis (Foto tomada en Canarias). b. Cephalopholis taeniops. c. Epinephelus costae (Foto T. Melo). d. Rypticus aff. saponaceus. e. Diplodus fasciatus (endémico). f. Diplodus sargus lineatus (endémico). g. Diplodus prayensis (endémico). h. Diplodus puntazzo (Foto tomada en Canarias). 


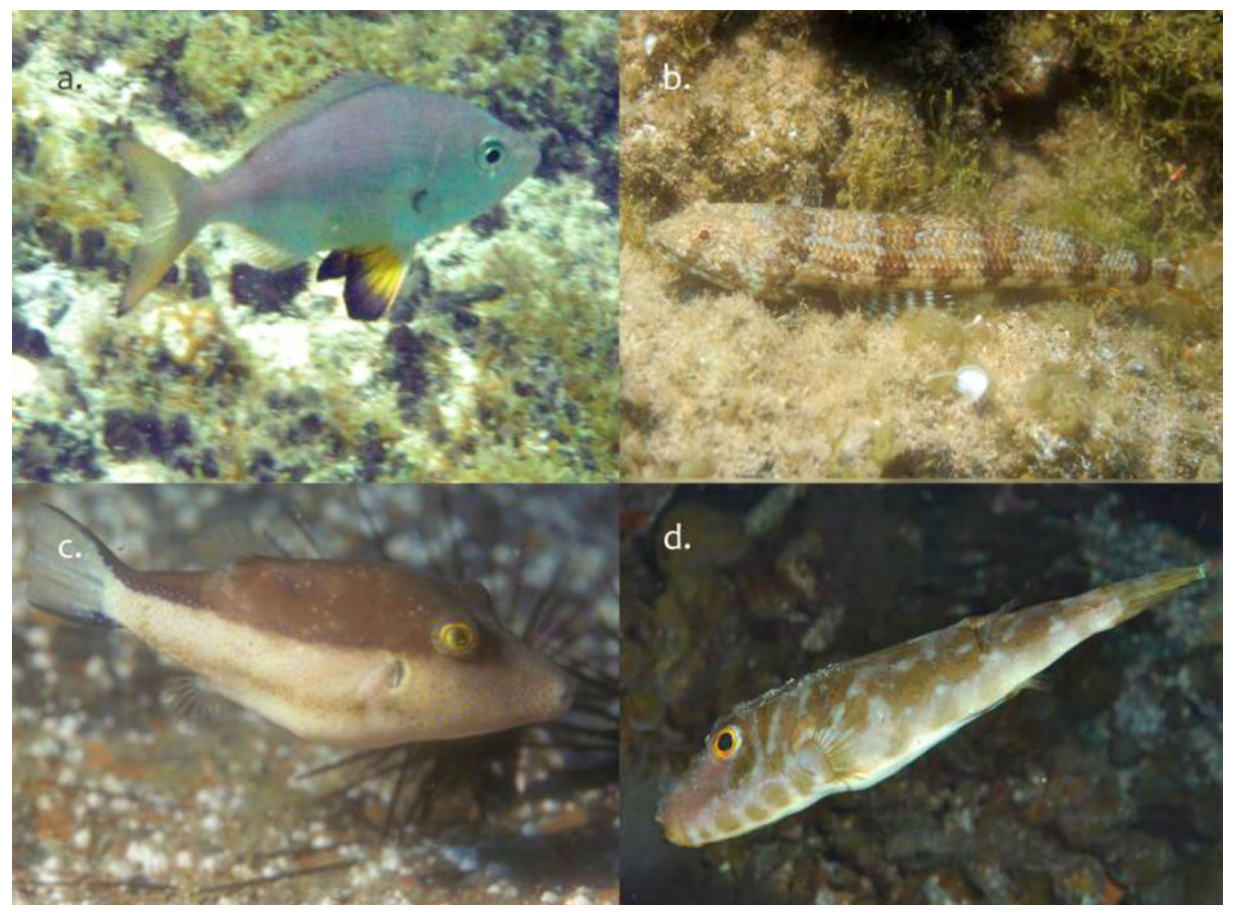

Lámina 7. a. Virididentex acromegalus (endémico). b. Synodus synodus (Foto tomada en Canarias). c. Canthigaster capistrata (Foto tomada en Canarias). d. Sphoeroides marmoratus (Foto tomada en Canarias). 